\title{
Construction of transplantable artificial vascular tissue based on adipose tissue-derived mesenchymal stromal cells by a cell coating and cryopreservation technique
}

\author{
Yoshiya Asano \\ Hirosaki University \\ Daisuke Okano \\ Hirosaki University \\ Michiya Matsusaki \\ Osaka University \\ Tetsuro Watabe \\ Tokyo Medical and Dental University \\ Yasuhiro Yoshimatsu \\ Niigata University \\ Mitsuru Akashi \\ Osaka University \\ Hiroshi Shimoda ( $\nabla$ hshimoda@hirosaki-u.ac.jp ) \\ Hirosaki University
}

\section{Research Article}

Keywords: HUVECs, NHDFs, ASCVT, hASCs

Posted Date: January 5th, 2021

DOI: https://doi.org/10.21203/rs.3.rs-135903/v1

License: (c) (i) This work is licensed under a Creative Commons Attribution 4.0 International License. Read Full License 
Construction of transplantable artificial vascular tissue based on adipose tissue-derived mesenchymal stromal cells by a cell coating and cryopreservation technique

Yoshiya Asano $^{1}$, Daisuke Okano ${ }^{1}$, Michiya Matsusaki², Tetsuro Watabe ${ }^{3}$, Yasuhiro Yoshimatsu $^{3,4}$, Mitsuru Akashi ${ }^{5}$, and Hiroshi Shimoda ${ }^{1,6^{*}}$

${ }^{1}$ Department of Neuroanatomy, Cell Biology and Histology, Graduate School of Medicine, Hirosaki University, Hirosaki, Japan

${ }^{2}$ Department of Applied Chemistry, Graduate School of Engineering, Osaka University, Osaka, Japan

${ }^{3}$ Department of Biochemistry, Graduate School of Medical and Dental Sciences, Tokyo Medical and Dental University (TMDU), Japan

${ }^{4}$ Division of Pharmacology, Graduate School of Medical and Dental Sciences, Niigata University, Niigata, Japan

${ }^{5}$ Building Block Science, Graduate School of Frontier Biosciences, Osaka University, Osaka, Japan

${ }^{6}$ Department of Anatomical Science, Graduate School of Medicine, Hirosaki University, Hirosaki, Japan

* Corresponding author: Hiroshi Shimoda, M.D., and Ph.D.

Telephone: +81-172-39-5004; Fax: +81-172-39-5006

E-mail: hshimoda@hirosaki-u.ac.jp 


\section{Abstract}

Prevascularized artificial three-dimensional (3D) tissues are effective biomaterials for regenerative medicine. We have previously established a scaffold-free 3D artificial vascular tissue from normal human dermal fibroblasts (NHDFs) and umbilical vein-derived endothelial cells (HUVECs) by layer-by-layer cell coating technique. In this study, we constructed an artificial vascular tissue constructed by human adipose tissue-derived stromal cells (hASCs) and HUVECs (ASCVT) by a modified technique with cryopreservation. ASCVT showed a higher thickness with more dense vascular networks than the 3D tissue based on NHDFs. Correspondingly, 3D-cultured ASCs showed higher expression of several angiogenesis-related factors, including vascular endothelial growth factor-A and hepatic growth factor, compared to that of NHDFs. Moreover, perivascular cells in ASCVT were detected by pericyte markers, suggesting the differentiation of hASCs into pericyte-like cells. Subcutaneous transplantation of ASCVTs to nude mice resulted in an engraftment with anastomosis of host's vascular structures at two weeks after operation. In the engrafted tissue, the vascular network was surrounded by mural-like structure-forming hASCs, in which some parts developed to form vein-like structures at four weeks, suggesting the generation of functional vessel networks. These results demonstrated that cryopreserved human cells, including hASCs, could be used directly to construct the artificial transplantable tissue for regenerative medicine. 


\section{Introduction}

Regenerative medicine by transplantation of artificial tissue is an ideal alternative to organ transplantation or when the treatment cannot be accomplished by usual medicine. Scaffold-free fabrication of artificial tissue by human cells is a trend in regenerative medicine regarding its low antigenicity and safety in transplantation and engraftment [1, 2]. Moreover, prevascularization of the artificial tissues has been attempted for medical applications [3-5], since blood circulation is critical for an effective engraftment of artificial tissues in order to prevent tissue degeneration due to low supply of nutrition and oxygen $[3,6]$. However, their application is still under development due to high costs, complication in fabrication, bio safety in human transplantation, and lack of well-established long-term grafts with vascular functions [7].

In our previous studies, three-dimensional (3D) artificial tissue with blood / lymphatic vascular network has been established using cell accumulation method with an extracellular matrix (ECM)-based layer-by-layer cell coating technique $[8,9]$. In this technique, the cultured human cells are coated with fibronectin and gelatin layers, resulting in a $10 \mathrm{~nm}$-thick coating ECM nano-films. Although these cells are free from artificial scaffolds, such as fibrin gel, they can form 3D artificial tissue within a short-term. For fabrication of vascular tissue, the fibronectin and gelatin-coated endothelial cells are cultivated between the layers of normal human dermal fibroblasts (NHDFs). They rapidly form a network of blood and/or lymphatic vessel-like tubules within a week $[9,10]$. To 
confirm their function, we have transplanted the 3D tissues with vascular networks, which were constructed using NHDFs and human umbilical vein-derived endothelial cells (HUVECs), into the subcutaneous tissue of nude mice. We found the engraftment of artificial vasculatures accompanied with their anastomosis to the host blood vessels and inflow of blood [3]. Moreover, the lymphatic vascular tissue constructed using NHDFs and human dermal lymphatic endothelial cells showed remodeling of the network with generation of blood vessel-like structures after engraftment into the back fascia or subcutaneous tissue of nude mice [4]. From these studies, we proposed the potency of the artificial vascular tissues constructed by the cell accumulation method in regenerative medicine [3, 4].

For the therapeutic application of artificial vascular tissues, use of autologous cells for transplantation is a reasonable strategy to avoid host rejection. Autologous mesenchymal stem / stromal cells (MSCs) are effective biomaterials for constructing tissue allowing successful engraftment [11]. In particular, adipose tissue-derived stromal cells (ASCs) are considered as an attractive biomaterial because the adipose tissue isolation from the donors or patients is minimally invasive. To date, much of studies for cellular transplantation of ASCs and their therapeutic applications have been reported, and it has been suggested that these cells induce tissue regeneration, differentiate to host tissue, and show immunomodulatory activities $[12,13]$. Because ASCs can induce blood and lymphatic vessel regeneration, these cells are suitable for the treatment of ischemic diseases and lymphedema [4, 14]. For transplantation, there are also tissue engineering efforts using ASCs to construct cell sheets [15] and 3D culture with artificial scaffolds $[16,17]$. Regarding the application of vascular tissue engineering, the co-cultivation of ASCs and endothelial cells in the fibrin matrix have been reported $[18,19]$. From these studies, the vascular tissue engineering will soon reach 
clinical applications. Scaffold-free strategies with simple and rapid technique would contribute to practical applications in regenerative medicine.

In the present study, we aimed to construct transplantable vascular tissue based on human ASCs (hASCs) by ECM-based cell accumulation method, with an addition of cryopreservation technique to elevate its availability in medical studies and clinical applications. For this purpose, the ECM nano-film-coated cells were cryopreserved using appropriate condition and used for tissue construction (refers as cryopreserved cell accumulation method; CP-CAM). By this method, we constructed the transplantable human vascular tissues based on hASCs, then evaluated in vitro characteristics and subcutaneous engraftment to nude mice, in comparison with those of fibroblast-based tissue as reported in our previous studies $[3,9]$.

\section{Results}

\section{Evaluation of the reagents and conditions for CP-CAM}

In order to adopt cryopreservation technique to the cell accumulation methods, we examined the effect of several cell freezing media on vessel network formation in artificial vascular tissue constructed by NHDFs and HUVECs (FbVT) (Fig. S1). In the control tissue (constructed by cell accumulation method without cryopreservation), the HUVECs formed human CD34-positive vascular network (Fig. S1 a) as similar to findings in previous studies $[3,4,10]$. However, cryopreservation using conventional cell freezing medium [Dulbecco's modified Eagle medium (DMEM) containing 10\% DMSO and 10\% FBS] resulted in the formation of a funicular structure by HUVECs without vascular network (Fig. S1 b). 
Moreover, the vascular network could not be formed when the cells were cryopreserved in two commercially available media, CELLBANKER1 (with serum) and STEM-

CELLBANKER (without serum) as shown in Fig. S1 c and d, respectively. On the other hand, the ECM nano-film-coated cells formed vascular network similar to the control group after cryopreservation in another commercially available medium, CultureSure Freezing Medium containing DMSO and recombinant human albumin without animal serum (termed as CSFM in this study) (Fig. S1 e). Due to these results, we also designed our original freezing medium containing DMSO and recombinant human albumin (refers to DMSOalbumin FM as described in Materials and Methods) and examined its cryopreserved effect on vascular network formation. The cells cryopreserved using this medium were able to form human CD34-positive vascular network as shown in Fig. S1 f. Although both CSFM and DMSO-albumin FM achieved the vascular formation, we obtained higher yields of living cells after thawing of cryopreserved stocks in CSFM (data not shown). Therefore, we further used CSFM for the CP-CAM in this study.

We evaluated the recovery of the living cells after cryopreservation for NHDFs, hASCs, and HUVECs (Fig. S2 a). The recovery rate of NHDFs was 70.6\% in the condition of non-coating and usage of conventional cell freezing medium (10\% DMSO and 10\% FBS in DMEM), and addition of ECM nano-film coating resulted no significant increase in the rate $(76.4 \%)$. On the other hand, the condition of ECM nano-film coating and usage of CSFM provided significantly higher recovery rate $(87.4 \%)$ than the other conditions. In this condition, hASCs and HUVECs showed relatively lower recovery rates (79.9\% and 69.0\%) than NHDFs, respectively, suggesting the different recovery rates depend on the cell types.

We further evaluated the recovery rates of NHDFs, hASCs, and HUVECs in CPCAM by using CSFM by focusing on the cell concentration and the length of the 
cryopreservation period (Fig. S2 b and c). From the results, we found that the recovery rates of each cell type were not significantly affected by the cell concentrations and cryopreservation periods.

\section{Artificial 3D vascular tissue fabricated by CP-CAM}

We analyzed the effect of CP-CAM on the vascular structures in FbVT prepared by ECM nano film-coated NHDFs and HUVECs without and with cryopreservation (refer to usual cell accumulation method and CP-CAM, respectively). On comparing these artificial tissues, we observed no significant differences between the vascular network in FbVT constructed by usual cell accumulation method and CP-CAM (Fig. S3 c and d). The tubular structure in FbVT fabricated by CP-CAM was also formed in the tissue sections as shown in Fig. S3 e, V. We found a few necrotic cells with swollen shape in the tissue suggesting a slight influence of cryopreservation (Fig. S3 e, black arrows).

In the transmission electron micrograph, the tissue consisted of fibroblasts, vascular structures constructed by HUVECs, and extracellular matrix (Fig. S3 f, Fb, V, and ECM, respectively). Formation of intercellular junctions between overlapping endothelial cells and basal lamina surrounding vascular structures were also found (Fig. S3 g, red arrow). These features corresponded to those in our previous study in which the vascular tissue was fabricated by usual cell accumulation method [3].

For further convenience, we also tested the cryopreservation mixing of the ECM nano-films coated NHDFs and HUVECs in ratio of 8:1. The seeding of thawed cells in nine layers on Transwell insert also resulted in the formation of vascular networks within four days as shown in Fig. S4. 
These results demonstrated that the artificial vascular tissues can be rapidly fabricated by CP-CAM, with almost equivalent structure as usual cell accumulation technique previously reported.

\section{Vascular network formation in hASC-based $3 D$ artificial vascular tissue fabricated by CP-}

\section{CAM}

Next, we fabricated artificial vascular tissue constructed by hASCs and HUVECs, termed as ASCVT, by CP-CAM, and the tissue structure was compared with that of the FbVT. As illustrated in Fig. 1 a, single layer of HUVECs was cultivated for four days between the bottom and top 4 layers of NHDFs or hASCs. The section of FbVT showed the distribution of vascular structure at the middle layer of the tissue (Fig. $1 \mathrm{a}, \mathrm{FbVT}$, arrowheads), similar to findings in previous reports $[9,10]$. On the other hand, ASCVT was about two times thicker than FbVT by abundant extracellular space between hASCs (Fig. 1 a, ASCVT and Fig. 1 b). The distribution of the vascular structure was not only in the middle part but also in the basal and top layer of the tissue (Fig. 1 a, ASCVT, arrowheads). Threedimensional analysis of the tissues clearly showed these distribution patterns of the vascular structure (Fig. 1 c, FbVT and ASCVT); the color labeling of vascular structure in FbVT and ASCVT along the depth of the tissue demonstrated the distribution of the network at the middle layer of FbVT and that at the top, middle, and bottom layers of ASCVT. The vertical distribution of the vascular structure showed additional peaks at top and bottom layers (Fig. 1 c, ASCVT, arrows) suggesting the three-dimensional elongation of the vessels in ASCVT. Furthermore, the vascular network density of ASCVT was higher than that of FbVT (Fig. 1 c). Further quantitative analysis of vascular network structures at the middle plane of FbVT 
and ASCVT confirmed these findings. The results in Fig. $1 \mathrm{~d}-\mathrm{f}$ showed a significantly higher score for total length, number of junctions, and vessel area in ASCVT than FbVT.

These results demonstrated that hASC-based vascular tissue was thicker and a more developed vascular structure than fibroblast-based vascular tissue.

\section{Analysis of angiogenesis-related factors profiles}

The results above demonstrated that the pro-angiogenic / vasculogenic activity of hASCs is more potent than NHDFs in the 3D culture. To confirm the key factors, the angiogenesis-related factors in the culture supernatants of 2D / 3D cultivated NHDFs and hASCs were examined by Proteome Profilar Human Angiogenesis Array Kit. We obtained the results of 55 factors as arrays of the dot immunoassays. Amongst them, the relative expression levels of thirteen factors were promoted in $3 \mathrm{D}$ cultivation compared to $2 \mathrm{D}$ cultivation as shown in Fig. 2. Three-dimensional culture of NHDFs showed an increased expression levels of eleven factors, including vascular endothelial growth factor-A (VEGFA) and hepatocyte growth factor (HGF), as shown in our previous study [9]. In the present study, we detected increased amount of other factors including factors such as angiogenin [20], amphiregulin [21], and PIGF [22], the mediators of endothelial cell migration and tubular structure formation such as CXCL16 [23], MCP-1 [24], MPP-8 [25], and prolactin [26], and the angiogenic regulatory factors such as DPPIV [27] and PF4 [28]. The 3D culture of hASCs also showed a similar profile of an increased expression of the angiogenesis-related factors. However, in particular, six factors were intensely promoted as indicated by the arrowheads in Fig. 2. Among these factors, an increase expression of FGF-7 (a keratinocyte growth factor) [29] was specifically found in the 3D culture of hASCs. These six factors 
would potentially contribute to the promotion of vascular network formation in ASCVT compared to FbVT.

In the artificial tissue fabricated by cell accumulation method, hypoxic condition has been shown in the tissue microenvironment [9]. Hence, the increase of the factors in 3D cultivated hASCs might be due to the hypoxic microenvironment. Therefore, we further analyzed the profile in 2D-cultured hASCs prepared under hypoxic condition. As shown in Fig. S5, the profile did not correspond with those of 3D cultivations. This result suggested an influence in the promotion of angiogenesis-related factors in ASCVT by some microenvironment conditions induced in 3D culture of ASCs rather than hypoxic condition.

\section{Pericyte-like differentiation of perivascular hASCs in ASCVT}

In the high magnification image of ASCVT, we observed the perivascular hASCs adjacent to the basement of the vascular endothelial cells (Fig. 3 a, arrowheads). On immunostaining, we found that the perivascular hASCs intensely expressed human CD90 (Fig. $3 \mathrm{~b}$ and c), and $\alpha \mathrm{SMA}$ (Fig. $3 \mathrm{~d}$ and e). In contrast, these intense staining were undetectable in the artificial tissue constructed by hASCs without vascular network, although slight staining was observed over the tissue (Fig. $3 \mathrm{f}$ and g). Next, we examined immunostaining for pericyte markers such as NG2 and desmin, and found positive reaction in the perivascular hASCs (Fig. $3 \mathrm{~h}-\mathrm{j}$ ). To confirm the perivascular localization of hASCs with pericyte-like characteristics, we constructed a vascular tissue consisting of NHDFs, EGFPlabeled hASCs, and HUVECs in the ratio of 7:1:1. Four days after construction, EGFPlabeled hASCs localized around and adhered to the vascular structure (Fig. 3 k, arrowheads, Fig. 31 and $\mathrm{m}$ ). 
We further observed the perivascular structure of ASCVT by transmission electron microscope (Fig. 4). The vascular structure constructed by HUVECs formed intercellular junctions (Fig. 4 a, red arrow), likewise FbVT (Fig. S3 g). The interaction between the perivascular hASCs and HUVECs was observed (Fig. 4 b); yellow arrow indicates the projection of hASC connected to the basal part of HUVEC, suggesting the formation of pegsocket structure which is commonly found between endothelial cells and pericytes [30, 31]. In addition, caveolae-like structures were observed in perivascular hASCs (Fig. 4 b, inset). We also observed abundant intracytoplasmic filaments in these cells (Fig. 4 c), which correspond to the immunostaining results of $\alpha \mathrm{SMA}$ in Fig. $4 \mathrm{~d}$ and e. In contrast, some hASCs apart from the vascular structure showed cytoplasmic ultrastructure resembling that of fibroblasts (Fig. 4 d).

These results demonstrated that perivascular hASCs were localized adjacent to the vascular structures, with intense expression of human CD90, showing pericyte-like differentiation.

\section{Subcutaneous transplantation of ASCVT in nude mice}

The artificial vascular network tissues (FbVT and ASCVT) were cut out from Transwell inserts at four days after their fabrication and subcutaneously transplanted to the nude mice (Fig. 5 a). After two weeks of transplantation, the engrafted tissues with host skin were collected, fixed and proceeded to tissue sectioning. The tissue sections were stained with hematoxylin and eosin (Fig. 5 b and d) or immunostained for human vimentin (Fig. 5 c and e). The engrafted FbVT had vascular structures that contained host erythrocytes (Fig. 6 b, inset) as reported in previous studies [3]. Immunostaining for human vimentin indicated the 
area of the graft (Fig. 5 c), and its average thickness was $66.95 \mu \mathrm{m}$ (Fig. 5 f). On the contrary, the engrafted ASCVT generated significantly thicker tissue than FbVT (Fig. 5 e and f). The average thickness was $137.14 \mu \mathrm{m}$, approximately two times that of the FbVT (Fig. $5 \mathrm{f}$ ). In the engrafted ASCVT, capillary- or small venule-like vascular structures were distributed in the abundant stroma (Fig. 5 g) forming dense collagen fiber as shown by Masson-Goldner staining (Fig. 5 h). The vascular structures contained the host erythrocytes (Fig. $5 \mathrm{~g}$ and h, high magnification), which demonstrated the anastomosis to the host circulation system. The double immunostaining for human CD34 and mouse / human CD31 showed that the mosaic structure consists of human and mouse endothelial cells suggesting the anastomosed structures at periphery of the graft area (Fig. $5 \mathrm{i}-\mathrm{k})$.

These results suggested that ASCVT constructed by CP-CAM subcutaneously engrafted the connective tissue containing the vessels linked to host blood flow.

\section{Vascular structure of subcutaneously engrafted ASCVT in nude mice}

We further observed the mural structure of engrafted vessels in ASCVT by immunohistochemistry. After two weeks of transplantation, the human CD90-positive cells surrounded the vessels in the engrafted tissue (Fig. 6 a) as well as $\alpha$ SMA-positive cells in the serial section (Fig. 6 b). These mural cells were also positive for NG2 (Fig. 6 c), corresponding to the presence of $\alpha$ SMA (Fig. $6 \mathrm{~d}-\mathrm{f}$ ), and desmin (Fig. $6 \mathrm{~g}$ ). In the electron micrograph of the engrafted vessels, multiple mural cells localized around the endothelial tubules (Fig. 6 h). Like the normal small vessels or capillaries, the basal part of endothelial cells and mural cells exhibited peg-socket structure in which the endothelial cells and mural cells can be connected (Fig. 6 i, arrow) [30, 31]. 
We also subcutaneously transplanted the vascular tissue consisting of NHDFs, EGFP-labeled hASCs, and HUVECs in the ratio of 7:1:1, and analyzed the engrafted tissue (Fig. S6 a - c). Almost every EGFP-labeled hASCs localized around human CD34-positive vessels (Fig. S6 b and c), demonstrating that hASCs in the graft participated in constructing the mural structure in the artificial vessels.

From these observations, we suggested that the pericyte-like differentiated cells from hASCs in ASCVT, were retained in the engrafted tissue and may act as mural cells of the vessels.

Moreover, in order to evaluate the long-term engraftment of ASCVT, we performed histological analysis of the graft four weeks after transplantation (Fig. 7). In this experiment, we used EGFP-labeled HUVECs for the fabrication of ASCVT. Although there was a replacement in some parts of the graft tissue by host tissue, we observed engrafted human blood vessels containing the host erythrocytes (Fig. $7 \mathrm{a}-\mathrm{f}$ ). Fig. $7 \mathrm{a}$ and b demonstrate vein-like vessels with approximately $50 \mu \mathrm{m}$ in diameter. These vessels showed positive immunostaining for human CD34 at endothelium. The vessels also showed staining for $\alpha \mathrm{SMA}$, human CD90 and NG2 at the mural structure constructed by $2-3$ layers of mural cells (Fig. $7 \mathrm{c}-\mathrm{e}$ ). Supporting the growth of vessels, the expression of Ki67, a marker of cellular proliferation, was detected in endothelial cells and mural cells in the engrafted tissue (Fig. S7). Moreover, some of the endothelial cells showed EGFP-positive (Fig. 7 f) proving that these cells derived from the artificial vessels constructed by HUVECs.

These results suggested that the transplanted ASCVT fabricated by CP-CAM could be retained in the host tissue for at least four weeks and could be constructed in the hierarchal vascular networks developing large size vessels, and connect to the host circulation. 


\section{Discussion}

Efficient cryopreservation techniques for the stem cells or fabricated tissues will enable timesaving and convenient use in medical studies and clinical applications. Due to this, several cryopreservation techniques of the stem cells including ESC, iPSC, and mesenchymal stem / stromal cells, as well as cryopreservation of fabricated tissues such as cell sheets and spheroids have been reported [32 - 34]. In the present study, we developed CP-CAM for construction of transplantable artificial tissues with a process of cell cryopreservation. In our evaluation of cell cryopreservation media for CP-CAM, CSFM which is a commercially available cell-freezing medium containing albumin, provided the most effective results of vascular network formation. Our designed cryopreservation medium (DMSO-albumin FM) containing recombinant human albumin also resulted in an efficient formation of vascular network by CP-CAM, suggesting that the albumin potentially plays a role in the preservation of vascular network-constructing capability of the frozen cells.

Albumin has been used for cell or tissue cryopreservation as the stabilizer of cell surface proteins in previous studies $[35,36]$. Although the detail of mechanisms is unclear, it was considered that the albumin may stabilize the ECM nano-films at cell surface in cryopreservation. CSFM provided better results than DMSO-albumin FM, suggesting that more factors in CSFM might support the preservation and tissue construction.

The CP-CAM is rapid and convenient for medical studies and clinical applications of artificial vascular tissue in regenerative medicine. After thawing of the cells from frozen stocks, the vascular tissue can be constructed within 4 days, that is the most time-saving 
compared to the other previous reports [5]. However, it should be noted that the recovery rates after cryopreservation were different among the tested cell types. Therefore, cryopreservation is preferable for some cell types with high recovery rates, combining with other cell types prepared without cryopreservation. In comparison among various conditions in CP-CAM, the recovery rates of the cells after thawing were not affected by cell concentrations and periods of cryopreservation. These results indicated the convenience of CP-CAM for experiments or therapeutic applications with flexible arrangement of the cells that are preserved in large scale for long-term periods.

By using CP-CAM, we constructed the artificial vascular tissue based on hASCs (ASCVT) and compared its structure to the tissue constructed based on human fibroblasts (FbVT). ASCVT had a thicker tissue structure and developed a denser vascular network than FbVT. Therefore, we expected more abundant production of angiogenesis-related factors by 3D-cultured ASCs. As shown in the protein profile analysis of culture supernatant, we found an intense expression of six factors by 3D-cultured hASCs. Excluding for PF4 as an angiostatic factor [29], the other five factors (VEGF-A, HGF, prolactin, MCP-1, and FGF-7) are pro-angiogenic / vasculogenic [24, 26, 37, 38]. Although the promoted expression of VEGF-A and HGF in 3D cultivation of fibroblasts was reported in our previous study [9], we found a more potent promotion of their expression in 3D cultivation of hASCs. For other factors, prolactin induces angiogenesis through endothelial cell migration [26], MCP-1 induces VEGF-A expression and tubular formation of endothelial cells, and FGF-7 is a potent inducer of angiogenesis / vasculogenesis [29, 39]. From these results, 3D-cultured hASCs by CP-CAM were shown to promote the expression of angiogenesis-related factors and provided the inductive condition for construction of artificial vascular network formation. 
In the ASCVT, we found the pericyte-like characteristics in perivascular cells. In these cells, we observed an intense expression of human CD90 and pericyte markers such as $\alpha \mathrm{SMA}, \mathrm{NG} 2$, and desmin [40], suggesting the differentiation of hASCs is possibly promoted by the interaction to HUVECs. The ASCs showing pericyte-like characteristics has been reported in the vascular morphogenesis by high-density cultivation of ASCs, which were considered to differentiate by the cellular interaction between heterotypic subpopulations [41]. Likewise, another study demonstrated that ASCs co-cultivated with endothelial cells in fibrin gel showed pericyte-like characteristics supporting and stabilizing the vascular structures [42]. In the adipose tissue, the ASCs distribute at perivascular areas [43], and they are sometimes regarded as equivalent to pericytes because of the common markers and cellular characteristics $[44,45]$. Recent studies indicated that ASCs show pericytic phenotype promoted by NOTCH2 in the interaction between vascular endothelial cells [46]. Another study also indicated that hASCs act as pericytes and immunomodulatory functions [47]. In the present study, the hASCs showed pericyte-like differentiation under the presence of vascular structures. However, not all perivascular ASCs showed this phenomenon, indicating that the hASC subpopulation non-capable of pericyte-like differentiation may exist.

Along this differentiation, the pericyte-like cells showed intense CD90 expression. It was reported that the adventitial pericyte progenitor with MSC-like phenotype expressed CD90 with pericyte markers [48]. In addition, human lung tissue contained the pericyte-like subtype of MSCs with CD90 and pericyte marker expression [49]. Thus, it was considered that the same perivascular behavior of hASC subpopulations would be reproduced in our present study. 
CD90 is one of the common markers for MSCs including ASCs [50]. Although its participation in self-renewal and differentiation of MSCs have been reported, the biological function of CD90 remains unclear [50]. Moraes et al. reported that knockdown of CD90 in MSC reduced the stemness guard of MSCs, enabling further differentiation when the specific stimuli are present [51]. For further analysis of perivascular MSC differentiation including the molecular mechanisms of CD90, the ASCVT has a high potential to be used as experimental tool.

The subcutaneous transplantation of ASCVT suggested their engraftment with an anastomosis to the host vessels and blood flow similar to the transplantation of FbVT [4]. The development of thicker tissues and dense vessel networks demonstrated prosperous tissue organization. To date, the induction of the tissue regeneration and angiogenesis by cellular transplantation of ASCs have been reported for therapeutic applications [52]. Our study showed that the scaffold-free 3D artificial vascular tissue based on hASCs could also promote both in vitro and in vivo tissue construction and vascular formation. The enhanced expression of pro-angiogenic factors shown in 3D cultivation of hASCs may contribute in these activities during in vivo processes.

Furthermore, we noticed the formation of perivascular mural-like structures by hASCs in the engrafted tissue. These structures exhibited pericyte- or vessel smooth muscle cell (SMC)-like characteristics with expression of NG2, $\alpha$ SMA, and desmin. Previously published data showed that different stimuli or co-culture methods induce ASCs to differentiate into pericytes or SMCs in vitro $[41,46,53]$. However, this differentiation in engrafted artificial tissue in vivo has never been reported. Our results clearly demonstrated that hASC-based perivasculary assembled and differentiated to form mural-like structures in engrafted ASCVT, suggesting formation of microcirculation with functional blood vessels. It 
is considered that enhanced pro-angiogenic activity and potency of hASCs in ASCVT developed in vitro would lead to the successful engraftment with structural maturation. Moreover, the maturation of engrafted vascular structure oriented from human cells was shown as development of blood vessels with large diameter in long-term periods after transplantation in our study. This result also suggested the development of functional vascular structure by transplantation of scaffold-free artificial vascular tissue. It has been reported that coverage of the vessels by mural structure and their interaction is necessary for vascular stabilization and further development $[40,54]$. Thus, the mural structure derived from hASC might promote the maturation of the blood vessels in transplanted tissue.

From our results by CP-CAM, the ASC-based vascular tissue using patient own cells can be used to enhance effective neovascularization. In this study, we used HUVECs were used as the source of vascular structure. For therapeutic applications, the vascular endothelial cells differentiated from the stem cells are possibly used instead of HUVECs [55, 56]. The endothelial progenitor cells derived from peripheral blood or umbilical blood are also available $[57,58]$. We have already confirmed the potential of human umbilical bloodderived endothelial precursor cells by our artificial vascular tissue-constructing technique (unpublished data). Endothelial cells from these sources will enable to construct transplantable artificial vascular tissues as materials for regenerative medicine of ischemic diseases such as ischemic heart disease, ischemic stroke, and chronic limb ischemia [59 - 61]. For this purpose, further analysis and assessment of vascular function, engraftment persistence, and safety in clinical usage are needed.

In conclusion, CP-CAM is capable of rapid construction of pre-vascularized artificial tissue using hASCs. The ASCVT was subcutaneously transplantable and formed 
hierarchal vascular networks alongside the engraftment. Our results demonstrated that cryopreserved human cells are able to construct the artificial transplantable tissue in shortterm, and the patient's own cells including ASCs are candidates for regenerative medicine. Furthermore, ASCVT will be a potent tool for studying perivascular differentiation of mesenchymal stem cells in vitro and in vivo models. The understanding of the intercellular communication and differentiation by using these models will lead to uncover the unknown mechanisms governing mesenchymal cell recruitment and differentiation alongside angiogenesis in development and regeneration.

\section{Methods}

\section{Cell culture, reagents, and animals}

Primary cultured NHDFs, neonatal hASCs and HUVECs were purchased from Lonza (Walkersville, MD). Each cell type was derived from single donor. NHDFs were cultured in Dulbecco's modified Eagle medium (DMEM, Wako Pure Chemical, Osaka, Japan) containing 10\% fetal bovine serum (FBS, SAFC Biosciences; Lenexa, KS) under 5\% $\mathrm{CO}_{2}$ at $37^{\circ} \mathrm{C}$ as described in our previous study [4]. For cultivation of HUVECs, Endothelial Growth Medium-2 (Lonza; Walkersville, MD) was used as described previously [3]. For cultivation of hASCs, Mesenchymal Stem Cell Growth Medium 2 (Takara Bio Inc., Kusatsu, Japan) was used. The reagents and animals were prepared according to our previous studies $[3,4]$ with some additions. Bovine plasma-derived fibronectin (FN) and porcine skin gelatin

(G) were purchased from Sigma-Aldrich (St. Louis, MO) and Wako Pure Chemical Industries, Ltd. (Osaka, Japan), respectively. Transwell inserts with porous polyester bottom 
(pore size: $0.4 \mu \mathrm{m}$ ) for 24 -well culture plate were purchased from CORNING Inc. (New York, NY). Female nude mice (BALB/cAJcl-nu) at six weeks of age were purchased from CLEA Japan, Inc. (Tokyo, Japan). Mouse anti-human vimentin monoclonal antibody (cat. No. M7020), mouse anti-human CD31 monoclonal antibody (cat. No. M8023), mouse antihuman CD34 monoclonal antibody (cat. No. M7165) were purchased from DAKO (Glostrup, Denmark). Mouse anti-alpha smooth muscle actin ( $\alpha$ SMA) monoclonal antibody (cat. No. ab40865), rabbit anti-human CD31 polyclonal antibody (cat. No. ab134168), and rabbit antimouse and human CD31 polyclonal antibody (cat. No. ab28364) were purchased from Abcam (Austin, TX). Rabbit anti-human CD90 polyclonal antibody (cat. No. AC-0054RUO) was purchased from EPITOMICS (Burlingame, CA). Rabbit anti-NG2 polyclonal antibody (cat. No. AB5320) was purchased from Merck Millipore (Darmstadt, Germany). Rabbit Ki67 polyclonal antibody (cat. No. 27309-1-AP) and rabbit anti-desmin polyclonal antibody (cat. No.16520-1-AP) were purchased from Proteintech (Rosemont, IL). Alexa Fluor 594conjugated goat anti-mouse IgG (cat. No. A11032) and Alexa Fluor 488-conjugated goat anti-rabbit IgG (cat. No. A11034) were purchased from Invitrogen (Carlsbad, CA). DAPI solution was purchased from Wako Pure Chemical (Osaka, Japan). Protein Profiler Human Angiogenesis Array Kit (cat No. ARY007) was purchased from R \& D Systems (Minneapolis, MN). All the animal experiments in this study were approved by the Animal Research Committee at Hirosaki University and were conducted according to the Guidelines for Animal Experimentation, Hirosaki University. Mice were maintained under controlled light (12-h light : dark cycle) and temperature $\left(21^{\circ} \mathrm{C}\right)$ conditions.

\section{EGFP labeling of hASCs and HUVECs by lentiviral transfection}


The experimental procedures were approved by the Genetically Modified Organisms Safety Committee of Hirosaki University (registration number: 16S001-1) and Tokyo Medical and Dental University (registration number: G2019-026C). For the fluorescence labeling of hASCs and HUVECs, GFP-bearing CS-CDF-CG-PRE lentiviral plasmid was used [62]. The 293FT cells were co-transfected with the expression plasmids and packaging plasmids (pCMV-VSV-G-RSV-Rev and pCAG-HIVgp) using Lipofectamine 2000 (11668019; Thermo Fisher Scientific). The viral supernatants were collected $48 \mathrm{~h}$ after transfection. For viral infection, $5.0 \times 10^{4}$ hASCs or HUVECs per well in 12-well tissue culture plates were infected with lentiviral particles.

\section{Construction of 3D artificial human vascular network tissue}

Using a cell accumulation method by layer-by-layer cell coating technique, we constructed multi layered 3D tissue as previously described [8-10, 63]. Briefly, cultured cells (NHDFs and hASCs within passage eight or HUVECs within passage six were collected by trypsinization, suspended in $0.04 \mathrm{mg} / \mathrm{mL} \mathrm{FN}$ in $50 \mathrm{mM}$ Tris- $\mathrm{HCl}$ buffer at $\mathrm{pH} 7.4$, and then incubated for $1 \mathrm{~min}$ with gentle rotation. Subsequently, the cells were treated with $0.04 \mathrm{mg} /$ $\mathrm{mL}$ of $\mathrm{G}$ in $50 \mathrm{mM}$ Tris- $\mathrm{HCl}$ buffer. Nine steps of coating (alternate $\mathrm{FN}$ and $\mathrm{G}$ coating) were performed to construct $10 \mathrm{~nm}$-thick ECM nano-film on each cell type $[8,9]$.

The coated cells were suspended in DMEM containing 10\% FBS and then seeded on Transwell inserts, which were placed in 24 -well cell culture plate containing the same medium. To construct the vascular tissue, four bottom layers of NHDFs or hASCs, a single layer of HDLECs, and four top layers of NHDFs or hASCs were applied daily, and the tissues were cultured in DMEM containing $10 \% \mathrm{FBS}$ at $37^{\circ} \mathrm{C}$ with $5 \% \mathrm{CO}_{2}$ for 5 days. Alternatively, NHDFs or hASCs and HUVECs were mixed in the cell number ratio of $8: 1$, 
seeded on Transwell inserts, and cultured in DMEM containing $10 \%$ FBS at $37^{\circ} \mathrm{C}$ with $5 \%$ $\mathrm{CO} 2$ for 5 days. In order to confirm the formation of the vascular network, the whole of fabricated 3D tissue was immunostained for human CD31 or human CD34 according to the method described below.

In this study, the artificial vascular tissues constructed by NHDFs / HUVECs and hASCs / HUVECs are termed FbVT and ASCVT, respectively.

\section{Cryopreserved cell accumulation method (CP-CAM)}

ECM nano-film-coated cells prepared by the method as described in 2.3 were cryopreserved using several cell freezing media as shown in Fig. S3 a. After the coating, the cells were collected by centrifugation at $1000 \mathrm{rpm}$ for $5 \mathrm{~min}, 15^{\circ} \mathrm{C}$, then resuspended in a serum-free cell freezing medium (CultureSure Freezing Medium; Fujifilm Wako, Osaka, Japan), termed as CSFM in this study, at the concentration of $5 \times 10^{5}-8 \times 10^{6}$ cells $/ \mathrm{ml}$ and placed on ice. The other four cryopreservation media: conventional cell freezing medium [DMEM containing 10\% FBS and 10\% dimethyl sulfoxide (DMSO)], CELLBANKER 1 (Takara Bio Inc.), STEMCELLBANKER (Takara Bio Inc.), and our originally designed cell freezing medium [DMEM containing $5 \mathrm{mg} / \mathrm{ml}$ of recombinant human albumin expressed in plants (Fujifilm Wako, Osaka, Japan) and 10\% DMSO], termed as DMSO-albumin FM, were also examined. The cell suspensions were aliquoted in the cryovials, kept at $-80^{\circ} \mathrm{C}$ overnight, and then stocked in liquid nitrogen.

In order to construct artificial tissue, the cryopreserved cells were thawed in the warm water at $37^{\circ} \mathrm{C}$ for two minutes, resuspended in DMEM without the serum, and centrifuged at $1000 \mathrm{rpm}$ for $5 \mathrm{~min}, 15^{\circ} \mathrm{C}$ as illustrated in Fig. S3 b. Then the cells were 
washed with DMEM containing 10\% fetal bovine serum and resuspended in the same medium with appropriate concentrations to fabricate the artificial tissue as described in 2.3.

\section{Analysis of the angiogenesis-related factors profile}

After cultivation of NHDFs and hASCs with suitable media, two-dimensional (2D) and $3 \mathrm{D}$ cultures were performed. For the $2 \mathrm{D}$ culture, $8 \times 10^{5}$ of NHDFs or hASCs without ECM nano-film were seeded onto $3 \mathrm{~cm}$-culture dishes with $2.3 \mathrm{ml}$ of DMEM and incubated for 24 hours under $5 \% \mathrm{CO}_{2}$ at $37^{\circ} \mathrm{C}$. For the $3 \mathrm{D}$ culture, $8 \times 10^{5}$ of NHDFs or hASCs with ECM nano-film were seeded on Transwell inserts and incubated with $2.3 \mathrm{ml}$ of DMEM for 24 hours under $5 \% \mathrm{CO}_{2}$ at $37^{\circ} \mathrm{C}$. Then, the culture supernatants were collected from $2 \mathrm{D} / 3 \mathrm{D}$ cultures (NHDFs or hASCs) and used to analyze the angiogenesis-related factors profile. The expression of 55 factors was analyzed using Proteome Profiler Human Angiogenesis Array Kit (R\&D Systems) according to the manufacturer's protocol. The quantitative analysis of detected factors was performed by using FIJI software (https://imagej.net/Fiji). The duplicated blots were obtained per factor, and their averaged scores were calculated.

\section{Transplantation of 3D artificial human vascular network tissue}

Transplantation of the artificial vascular tissue was performed according to the method described in the previous studies $[3,4]$. Briefly, the fabricated artificial tissues were collected from the Transwell inserts by cutting the bottom polyester membranes and kept in DMEM at $37^{\circ} \mathrm{C}$ until transplantation into the subcutaneous tissue of nude mice. Under anesthesia, the dorsal skin of the mice was cut to a length of $1.5 \mathrm{~cm}$, and the artificial tissues were then inserted subcutaneously, facing the artificial tissue up to the skin tissue. The 
wound was then immediately closed using silk sutures. For immunosuppression, cyclosporin (Neoral, Novartis, Rueil-Malmaison, France) was added to drinking water (120 mg / L), one week before and all along the engraftment period as previously reported [64]. After 2 weeks and 4 weeks of transplantation, the mice were euthanized, then the dorsal skin including the graft was collected for histological examination.

\section{Light and fluorescence microscopy}

The fabricated artificial vascular tissues in the Transwell inserts were fixed with $4 \%$ paraformaldehyde in $0.1 \mathrm{M}$ phosphate buffer $\left(\mathrm{pH}\right.$ 7.4) for overnight at $4{ }^{\circ} \mathrm{C}$ and collected from the Transwell inserts by cutting the bottom polyester membranes. In order to achieve whole-mount immunostaining, the tissues were treated with $0.3 \%$ Triton $\mathrm{X}-100$ in $0.1 \mathrm{M}$ phosphate buffer at $4^{\circ} \mathrm{C}$ for 3 days. Then, the tissues were stained according to the method described in our previous studies $[3,4,10]$. The specimens were observed using a confocal microscope Nikon C2 (Nikon, Tokyo, Japan). For the histological analysis, the fixed artificial tissues embedded in paraffin, and then $5 \mu \mathrm{m}$ thick serial tissue sections were prepared. The engrafted tissues collected from mouse dorsal skin were also fixed in 4\% paraformaldehyde in 0.1 M phosphate buffer ( $\mathrm{pH} 7.4$ ) and embedded in paraffin. For Masson-Goldner staining, the deparaffinized sections were treated with acid fuchsin solution, phosphomolybdic acidOrange G solution, and Light Green solution using the conventional method, and then mounted using a cover slip. For immunohistochemistry, antigen retrieval was performed by boiling the deparaffinized sections twice in $10 \mathrm{mM}$ citric acid (pH 6.0) using a microwave oven at $500 \mathrm{~W}$ for $5 \mathrm{~min}$ each. The sections were then treated with $3 \%$ normal goat serum (Wako) in $0.1 \mathrm{M}$ phosphate buffer (pH 7.4) containing $0.05 \%$ Tween 20 for $1 \mathrm{~h}$ at room temperature, and subsequently incubated overnight at $4{ }^{\circ} \mathrm{C}$ with the primary antibodies. For 
dark field microscopy, the sections were visualized by incubation with fluorescence-labeled secondary antibodies, goat anti-mouse IgG conjugated with Alexa-Fluor 594 or goat antirabbit IgG conjugated with Alexa-Fluor 488. The specimens were observed using a light microscope BX-50 (Olympus, Tokyo, Japan) or a fluorescence microscope BZ-X700 (Keyence, Tokyo, Japan).

\section{Transmission electron microscopy}

The artificial vascular tissues and engrafted tissues were fixed in $2.5 \%$ glutaraldehyde and $2 \%$ paraformaldehyde in $0.1 \mathrm{M}$ phosphate buffer $(\mathrm{pH} 7.4)$ at $4^{\circ} \mathrm{C}$. The tissues were cut into pieces $(1 \mathrm{~mm} \times 1 \mathrm{~mm})$ and post-fixed in $1 \%$ osmium tetroxide in $0.1 \mathrm{M}$ phosphate buffer. The tissues were then dehydrated and embedded in Epon 812 (Nisshin EM, Tokyo, Japan), followed by section preparation with $70 \mathrm{~nm}$-thickness using an ultramicrotome (REICHERT ULTRACUT S, Leica, Wetzlar, Germany). The sections were then stained with 4\% uranyl acetate and lead stain solution (Sigma Aldrich, St. Louis, MO) by the conventional method, and observed using a transmission electron microscope (JEM1200, JEOL, Tokyo, Japan).

\section{Quantitative analysis of image data}

Areas of human CD34-positive vascular network in immunofluorescent photographs were extracted by using Photoshop software (Adobe, San Jose, CA) and their total length, branches and areas were quantified by using Image $\mathrm{J}$ software (https://imagej.nih.gov/ij/) and Angiogenesis Analyzer (http://image.bio.methods.free.fr/ImageJ/?Angiogenesis-Analyzer-for-ImageJ\&lang=en). Three-dimensional analysis of vascular network was also performed by using FIJI software 
(https://imagej.net/Fiji). Statistical analysis of these data was performed using one-way ANOVA $(\mathrm{n}=5) . P$-values of less than 0.05 were considered statistically significant.

\section{References}

1. Arai, K. et al. Fabrication of scaffold-free tubular cardiac constructs using a Bio-3D printer. PLoS One. 13, e0209162 (2018).

2. Ntege, E. H., Sunami, H. \& Shimizu, Y. Advances in regenerative therapy: A review of the literature and future directions. Regen Ther. 14, 136-153 (2020).

3. Asano, Y., Shimoda, H., Okano, D., Matsusaki, M. \& Akashi, M. Transplantation of three-dimensional artificial human vascular tissues fabricated using an extracellular matrix nanofilm-based cell-accumulation technique. J. Tissue Eng. Regen. Med. 11, 1303-1307 (2017).

4. Asano, Y., Shimoda, H., Matsusaki, M. \& Akashi, M. Transplantation of artificial human lymphatic vascular tissues fabricated using a cell-accumulation technique and their engraftment in mouse tissue with vascular remodeling. J. Tissue Eng. Regen. Med. 12, e1501e1510 (2018). 
5. Wang, Z., Mithieux, S. M. \& Weiss, A. S. Fabrication Techniques for Vascular and Vascularized Tissue Engineering. Adv. Healthc. Mater. 8, e1900742 (2019).

6. Klar, A. S. et al. Tissue-engineered dermo-epidermal skin grafts prevascularized with adipose-derived cells. Biomaterials. 35, 5065-5078 (2014).

7. Laschke, M. W. \& Menger, M. D. Prevascularization in tissue engineering: Current concepts and future directions. Biotechnol. Adv. 34, 112-121 (2016).

8. Matsusaki, M., Case, C. P. \& Akashi, M. Three-dimensional cell culture technique and pathophysiology. Adv. Drug. Deliv. Rev. 74, 95-103 (2014).

9. Nishiguchi, A., Matsusaki, M., Asano, Y., Shimoda, H. \& Akashi, M. Effects of angiogenic factors and 3D-microenvironments on vascularization within sandwich cultures. Biomaterials. 35, 4739-4748 (2014).

10. Asano, Y. et al. Ultrastructure of blood and lymphatic vascular networks in threedimensional cultured tissues fabricated by extracellular matrix nanofilm-based cell accumulation technique. Microscopy (Oxf). 63, 219-226 (2014).

11. Tavakoli, S. et al. Mesenchymal stromal cells; a new horizon in regenerative medicine. J Cell Physiol. 235, $9185-9210$ (2020) 
12. R. Dai, R., Wang, Z., Samanipour, R., Koo, K. I. \& Kim, K. Adipose-Derived Stem Cells for Tissue Engineering and Regenerative Medicine Applications. Stem Cells Int. 2016, 6737345 (2016).

13. Bajek, A. et al. Adipose-Derived Stem Cells as a Tool in Cell-Based Therapies. Arch. Immunol. Ther. Exp (Warsz). 64, 443-454 (2016).

14. Teraa, M., Gremmels, H., J Wijnand, J. G. \& Verhaar, M. C. Cell Therapy for Chronic Limb-Threatening Ischemia: Current Evidence and Future Directions. Stem Cells Transl. Med. 7, 842-846 (2018).

15. Takemura, S., Shimizu, T., Oka, M., Sekiya, S. \& Babazono, T. Transplantation of adipose-derived mesenchymal stem cell sheets directly into the kidney suppresses the progression of renal injury in a diabetic nephropathy rat model. J. Diabetes Investig. 11, 545553 (2020).

16. Hutton, D. L. et al. Tumor necrosis factor improves vascularization in osteogenic grafts engineered with human adipose-derived stem/stromal cells. PLoS One. 9, e107199 (2014).

17. Manavella, D. D. et al. Adipose tissue-derived stem cells in a fibrin implant enhance neovascularization in a peritoneal grafting site: a potential way to improve ovarian tissue transplantation. Hum. Reprod. 33, 270-279 (2018). 
18. Rohringer, S. et al. Mechanisms of vasculogenesis in 3D fibrin matrices mediated by the interaction of adipose-derived stem cells and endothelial cells. Angiogenesis. 17, 921-933 (2014)

19. Pill, K. et al. Microvascular Networks From Endothelial Cells and Mesenchymal Stromal Cells From Adipose Tissue and Bone Marrow: A Comparison. Front. Bioeng. Biotechnol. 6, 156 (2018).

20. Miyake, M., Goodison, S., Lawton A., Gomes-Giacoia, E. \& Rosser, C. J. Angiogenin promotes tumoral growth and angiogenesis by regulating matrix metallopeptidase-2 expression via the ERK1/2 pathway. Oncogene. 34, 890-901 (2015).

21. Wang, C. Q. et al. Amphiregulin enhances VEGF-A production in human chondrosarcoma cells and promotes angiogenesis by inhibiting miR-206 via FAK/cSrc/PKC $\delta$ pathway. Cancer Lett. 385, 261-270 (2017).

22. De Falco, S. The discovery of placenta growth factor and its biological activity. Exp. Mol. Med. 44, 1-9 (2012).

23. Isozaki, T., et al. Evidence that CXCL16 is a potent mediator of angiogenesis and is involved in endothelial progenitor cell chemotaxis : studies in mice with $\mathrm{K} / \mathrm{BxN}$ seruminduced arthritis. Arthritis Rheum. 65, 1736-1746 (2013). 
24. Niu J., Azfer, A., Zhelyabovska, O., Fatma, S. \& Kolattukudy, P. E. Monocyte chemotactic protein (MCP)-1 promotes angiogenesis via a novel transcription factor, MCP-1induced protein (MCPIP). J. Biol. Chem. 283, 14542-14551 (2008).

25. Fang, C. et al. An important role of matrix metalloproteinase- 8 in angiogenesis in vitro and in vivo. Cardiovasc. Res. 99 146-155 (2013).

26. Reuwer, A. Q. et al. Functional consequences of prolactin signaling in endothelial cells: a potential link with angiogenesis in pathophysiology?. J. Cell Mol. Med. 16, 2035-2048 (2012).

27. Lei, Y. et al. Dipeptidyl Peptidase-IV Inhibition for the Treatment of Cardiovascular Disease - Recent Insights Focusing on Angiogenesis and Neovascularization. Circ. J. 81, $770-776(2017)$

28. Lord, M. S., Cheng, B., Farrugia, B. L., McCarthy, S. \& Whitelock, J. M. Platelet Factor 4 Binds to Vascular Proteoglycans and Controls Both Growth Factor Activities and Platelet Activation. J. Biol. Chem. 292, 4054-4063 (2017).

29. Vadija, R. et al. Homology modeling and virtual screening studies of FGF-7 protein-a structure-based approach to design new molecules against tumor angiogenesis. J. Chem. Biol. 9, 69-78 (2016). 
30. Armulik, A., Abramsson, A. \& Betsholtz, C. Endothelial/pericyte interactions. Circ. Res. 97, 512-523 (2005).

31. Hayden, M. R., Yang, Y., Habibi, J., Bagree, S. V. \& Sowers, J. R. Pericytopathy: oxidative stress and impaired cellular longevity in the pancreas and skeletal muscle in metabolic syndrome and type 2 diabetes. Oxid. Med. Cell Longev. 3, 290-303 (2010).

32. Bissoyi, A., Pramanik, K., Panda, N. N. \& Sarangi, S. K. Cryopreservation of hMSCs seeded silk nanofibers based tissue engineered constructs. Cryobiology. 68, 332-342 (2014).

33. Pless-Petig, G., Knoop, S. \& Rauen, U. Serum- and albumin-free cryopreservation of endothelial monolayers with a new solution, Organogenesis. 14, 107-121 (2018).

34. Arai, K., Murata, D., Takao, S., Verissiomo, A. R. \& Nakayama, K. Cryopreservation method for spheroids and fabrication of scaffold-free tubular constructs. PLoS One. 15, e0230428 (2020).

35. Mirabel, C. et al. Stability enhancement of clinical grade multipotent mesenchymal stromal cell-based products. J. Transl. Med. 16, 291 (2018).

36. González Porto, S. A. et al. The addition of albumin improves Schwann cells viability in nerve cryopreservation. Cell Tissue Bank. 19, 507-517 (2018). 
37. Cao, R. et al. Hepatocyte growth factor is a lymphangiogenic factor with an indirect mechanism of action. Blood. 107, 3531-3536 (2006).

38. Neufeld, S., Planas-Paz, L. \& Lammert, E. Blood and lymphatic vascular tube formation in mouse. Semin. Cell Dev. Biol. 31, 115-123 (2014).

39. Gillis, P. et al. Keratinocyte growth factor induces angiogenesis and protects endothelial barrier function. J. Cell Sci. 112, 2049-2057 (1999).

40. Geevarghese, A. \& Herman, I. M. Pericyte-endothelial crosstalk: implications and opportunities for advanced cellular therapies. Transl. Res. 163, 296-306 (2014).

41. Hutton, D. L. et al. Vascular morphogenesis of adipose-derived stem cells is mediated by heterotypic cell-cell interactions. Tissue Eng. Part A. 18, 1729-1740 (2012).

42. Pill, K. et al. Microvascular Networks From Endothelial Cells and Mesenchymal Stromal Cells From Adipose Tissue and Bone Marrow: A Comparison. Front. Bioeng. Biotechnol. 6, 156 (2018).

43. Locke, M., Windsor, J. \& Dunbar, P. R. Human adipose-derived stem cells: isolation, characterization and applications in surgery. ANZ J. Surg. 79, 235-244 (2009). 
44. Geevarghese, A. \& Herman, I. M. Pericyte-endothelial crosstalk: implications and opportunities for advanced cellular therapies. Transl. Res. 163, 296-306 (2014).

45. Hajmousa, G. et al. Human adipose tissue-derived stromal cells act as functional pericytes in mice and suppress high-glucose-induced proinflammatory activation of bovine retinal endothelial cells. Diabetologia 61, 2371-2385 (2018).

46. Terlizzi, V., Kolibabka, M., Burgess, J. K., Hammes, H. P. \& Harmsen,M.C. The Pericytic Phenotype of Adipose Tissue-Derived Stromal Cells Is Promoted by NOTCH2. Stem Cells. 36, 240-251 (2018).

47. Hajmousa, G. et al. Human adipose tissue-derived stromal cells act as functional pericytes in mice and suppress high-glucose-induced proinflammatory activation of bovine retinal endothelial cells. Diabetologia. 61, 2371-2385 (2018).

48. Tigges, U., Komatsu, M. \& Stallcup, W. B. Adventitial pericyte progenitor/mesenchymal stem cells participate in the restenotic response to arterial injury. J. Vasc. Res. 50, 134-144 (2013).

49. Wang, L. et al. CD $90^{+} \mathrm{CD} 146^{+}$identifies a pulmonary mesenchymal cell subtype with both immune modulatory and perivascular-like function in postnatal human lung. Am. $J$. Physiol. Lung Cell Mol. Physiol. 318, L813-L830 (2020). 
50. Maleki, M., Ghanbarvand, F., Reza Behvarz, M., Ejtemaei, M. \& Ghadirkhomi, E. Comparison of mesenchymal stem cell markers in multiple human adult stem cells. Int. J. Stem Cells. 7, 118-126 (2014).

51. Moraes, D. A. et al. A reduction in CD90 (THY-1) expression results in increased differentiation of mesenchymal stromal cells. Stem Cell Res. Ther. 7, 97 (2016).

52. Feisst, V., Meidinger, S. \& Locke, M. B. From bench to bedside: use of human adiposederived stem cells. Stem Cells Cloning 8, 149-162 (2015).

53. Parvizi, M., Bolhuis-Versteeg, L. A., Poot, A. A. \& Harmsen, M. C. Efficient generation of smooth muscle cells from adipose-derived stromal cells by $3 \mathrm{D}$ mechanical stimulation can substitute the use of growth factors in vascular tissue engineering. Biotechnol. J. 11, 932-944 (2016).

54. Stratman, A. N. et al. Weinstein, Interactions between mural cells and endothelial cells stabilize the developing zebrafish dorsal aorta. Development. 144, 115-127 (2017).

55. Fontijn, R. D. et al. Adipose tissue-derived stromal cells acquire endothelial-like features upon reprogramming with SOX18. Stem Cell Res. 13, 367-378 (2014). 
56. Khan, S. et al. Fibroblast growth factor and vascular endothelial growth factor play a critical role in endotheliogenesis from human adipose-derived stem cells. J. Vasc. Surg. 65, 1483-1492 (2017).

57. Bayraktutan, U. Endothelial progenitor cells: Potential novel therapeutics for ischaemic stroke. Pharmacol. Res. 144, 181-191 (2019).

58. Kalka, C. et al. Transplantation of ex vivo expanded endothelial progenitor cells for therapeutic neovascularization. Proc. Natl. Acad. Sci. USA. 97, 3422-3427 (2000).

59. Fujita, Y. \& Kawamoto, A. Stem cell-based peripheral vascular regeneration. $A d v$. Drug Deliv. Rev. 120, 25-40 (2017).

60. Esteban-Garcia, N. et al. Neurorestoration Approach by Biomaterials in Ischemic Stroke. Front. Neurosci. 14, 431 (2020).

61. Hénon, P. Key Success Factors for Regenerative Medicine in Acquired Heart Diseases. Stem Cell Rev. Rep. 16, 441-458 (2020).

62. Akatsu, Y. et al. Fibroblast growth factor signals regulate transforming growth factor- $\beta$ induced endothelial-to-myofibroblast transition of tumor endothelial cells via Elk1. Mol.

Oncol. 13, 1706-1724 (2019). 
63. Nishiguchi, A., Yoshida, H., Matsusaki, M. \& Akashi, M. Rapid construction of threedimensional multilayered tissues with endothelial tube networks by the cell-accumulation technique. Adv. Mater. 23, 3506-3510 (2011).

64. Kökten, T. et al. Immunomodulation stimulates the innervation of engineered tooth organ. PLoS One. 9, e86011 (2014).

\section{Acknowledgements}

The authors thank Dr. Hiroyuki Miyoshi for the lentiviral vectors, Mr. Koji Noguchi and Mr. Daisuke Hatanaka for technical assistance and discussions. This work was supported by JSPS KAKENHI Grant Number 17K08506 and a grant from the 37th Karoji Memorial Fund for Medical Research (Grant A to Y. Asano, 2019).

\section{Author contributions}

Y.A. designed the studies, performed the experiments, interpreted the data, and wrote the paper. Y.A. also contributed to funding acquisition. D.O. performed EGFP labeling of culture cells by using lentiviral vectors and morphological analysis of the artificial tissues. M.M. and M.A. contributed to the methodology of layer by layer cell coating technique. T.W. and Y.Y. contributed to the construction and transfection of EGFP lentiviral vectors for 
labeling of culture cells. H.S. administrated the project, supervised the studies, and contributed to funding acquisition. All authors contributed to revision of the manuscript and have approved the submitted version.

\section{Competing interests}

The author(s) declare no competing interests.

\section{Data availability statement}

The data that support the findings of this study are available from the corresponding author (HS) upon request.

\section{Figure legends}

Figure 1: Comparison of the vascular tissues constructed by CP-CAM based on NHDFs and hASCs.

a: The vascular tissues were constructed by sequential pile of basal four layers of NHDF or hASC (B), middle single layer of HUVEC (V), and top four layers of NHDF or hASC (T). For four days, 3D cultivation of the piled cells resulted the formation of artificial tissues with 
vascular structures. The microscopic images of toluidine blue staining sections are shown (FbVT: vascular tissue based on NHDFs; ASCVT: vascular tissue based on hASCs). Arrowheads indicate the distribution of the vascular structures. b: Comparison of the thickness of FbVT and ASCVT. c: The integrated confocal 3D images of human CD34positive vascular networks in FbVT and ASCVT. Color bars denote the depth along z-axis of the tissue, and the vascular images colored according to the depth of the tissues. The graphs show the relative density distribution of the vascular structure along the depth of the FbVT and ASCVT. Hatched lines indicate the depth of middle vascular layer. The vascular network of ASCVT showed a more dense structure compared to that of FbVT, and spreads to the top and bottom layers indicated by multiple color of the vascular images. Accordingly, the additional peaks of vascular distribution appeared at top and bottom layers as indicated by red arrows. d - f: Quantitative analysis of vascular total length (d), number of junctions (e), and vessel area (f) in FbVT and ASCVT.

Figure 2: Profiles of angiogenesis-related factors in culture supernatants collected from

\section{D- and 3D-cultivated NHDFs and hASCs}

The culture supernatants were analyzed using Proteome Profiler Human Angiogenesis Array Kit (R\&D Systems). Among 55 angiogenesis-related factors, 13 factors increased in 3D cultivation compared to 2D cultivation of NHDFs or ASCs and are shown in these graphs (ND: not detected). Arrowheads: the factors specifically increased in the supernatant from 3D cultivation of ASCs.

\section{Figure 3: Perivascular differentiation of hASCs in ASCVT}


a: Highly magnified image of epon-embedded and sectioned ASCVT with toluidine blue staining. V: vascular structures. Perivascularly localized hASCs are observed (red arrowheads). b - g: Immunostaining for human CD34 (hCD34) / human CD90 (hCD90) (b, c, f) and human CD31 (hCD31) / aSMA (d, e, g). b - e: ASCVT. Vascular structures (V) are immunostained for hCD34 and hCD31. Perivascularly localized hASCs show intense immunostaining for hCD90 and $\alpha$ SMA (C and E). f and $\mathbf{g}$ : Artificial tissue constructed by hASCs without HUVECs. No vascular structures are found. A weak staining of hCD90 and aSMA is observed. $\mathbf{h}$ and $\mathbf{i}$ : Perivasculaly localized hASCs showing immunostaining for NG2 (yellow arrowheads). j: Perivasculaly localized hASCs showing immunostaining for desmin (yellow arrowheads). k - m: Addition of EGFP-labeled hASCs to fibroblast-based vascular tissue (NHDFs: hASCs: HUVECs = 7: 1: 1). k: Immunostaining of the paraffin section. hASCs with EGFP localized to perivascular area (yellow arrowheads). I: Highly magnified image of hatched box in k. EGFP-positive hASC connected to the vascular structure (V). m: 3D-constructed confocal image. EGFP-positive hASC surrounded the vascular structure like a pericyte. The nuclei of the cells were visualized by DAPI (blue color).

\section{Figure 4: Ultrastructure of perivascularly localized hASCs in ASCVT}

a: Electron micrograph of ASCVT. V: vascular structure constructed by HUVECs (redcolored). Interendothelial connecting junctions are observed (red arrow). Perivascularly localized hASCs are also shown. ECM: extracellular matrix is more abundant compared to FbVT in Figure 1 f. b: High magnified image of hatched box in a. The projections from hASC interact with HUVEC, suggesting the formation of peg-socket structure (yellow 
arrow). Inset shows further high magnified image of red hatched box containing caveola at cytoplasmic membrane. c: Another high magnified image of hatched box in a. The cytoplasm of perivascular hASCs shows filamentous structure that is considered as presence of actin filaments. Inset shows further high magnified image of red hatched box on cytoplasm. $\mathbf{d}$ : The image of hASCs locate far from vascular structures. The cytoplasm shows the fibroblast-like structure with abundant rough endoplasmic reticulum (rER) and mitochondria (mt).

\section{Figure 5: Subcutaneous transplantation of ASCVT to nude mice}

a: FbVT and ASCVT were cut out from the Transwell inserts and subcutaneously transplanted to back skin of nude mice. $\mathbf{b}$ - e: engrafted FbVT (b and c) and ASCVT ( $d$ and e) at two weeks after the transplantation. $\mathbf{b}$ and $\mathbf{d}$ : HE staining; $\mathbf{c}$ and $\mathbf{e}$ : immunostaining for human vimentin. The FbVT engrafts as connective tissue containing vascular networks with host blood (b, inset). The thickness of engrafted human-derived tissue is visualized by immunoreaction of human vimentin (c). The ASCVT also subcutaneously engrafted with vascular networks (d), and the thickness was higher than engrafted FbVT (e). f: Quantitative comparison of the graft thickness between FbVT and ASCVT. The ASCVT significantly shows higher thickness. g - k: High magnified images of engrafted ASCVT. g: HE staining. The graft contains the capillary- or small venule-like structures with host blood (V) and abundant stroma. h: Masson Goldner staining for serial section of g. The stroma stained for collagen fibers, indicating the construction of connective tissue. $\mathbf{i}$ - $\mathbf{k}$ : Immunostaining by using antibodies for human CD34 (red) and mouse / human CD31 (green). The engrafted vessels derived from HUVEC are positive for both antibodies (i and $j$ ). At the peripheral area of the graft, the vessels with staining by antibodies for mouse / human CD31 and partial 
staining by antibodies for human CD34 are observed ( $\mathrm{i}$ and $\mathrm{k}$ ) suggesting the anastomotic region between host and graft circulation. The nuclei of the cells in the dark field images were visualized by DAPI (blue color).

Figure 6: The vascular mural structure in the engrafted ASCVT at 2 weeks after the transplantation

a: Immunostaining for human CD34 (hCD34) and human CD90 (hCD90). Almost every engrafted and hCD34-positive vessels surrounded by hCD90-positive mural cells that putatively derived from hASCs. b: Immunostaining for human CD31 (hCD31) and $\alpha$ SMA in serial section of $\mathrm{A}$. The endothelial cells are positive for hCD31 in addition to hCD34. The mural cells are positive for $\alpha$ SMA. c: Immunostaining for hCD34 and NG2. The mural cells are positive for NG2 that is a marker of pericytes and vascular smooth muscle cells. $\mathbf{d}$ - $\mathbf{f}$ : double immunostaining for $\alpha$ SMA and NG2. The merged image indicated coexistence of aSMA and NG2 in the mural cells derived from hASCs. g: The presence of desmin, another marker of pericytes and smooth muscle cells, is confirmed in the mural cells. h: electron micrograph of the vessels in engrafted ASCVT. Er: erythrocytes; Ec: endothelial cells; Mu: mural cells. Stratifying mural cells surrounded the vessels. i: High magnified image of hatched box in h. Formation of peg-socket structure between endothelial cell and the mural cell is found (arrow). The nuclei of the cells in the dark field images were visualized by DAPI (blue color).

Figure 7: The blood vessels in the engrafted ASCVT at four weeks after the transplantation 
a - f: The engrafted ASCVT at four weeks after transplantation. The serial sections are used for each staining. a and b: HE staining. Enlarged vein-like vessels (V) are found in the graft. c - f: Immunostaining. The endothelium of vein-like vessel is positive for both mouse / human CD31 (m/hCD31) (c) and human CD34 (hCD34) (d and e). The mural cells show positive immunostaining for $\alpha$ SMA (c), human CD90 (hCD90) (d), and NGs (e), indicating that this structure derived from hASCs. f: Immunostaining for human vimentin and EGFP. The engrafted ASCVT was constructed by using HUVEC labeled by EGFP. The endothelial cells showed EGFP-positive immunostaining (arrows), demonstrating that the vascular structures in ASCVT engrafted and constructed the vein-like vessels surrounded by mural cells. The nuclei of the cells in the dark field images were visualized by DAPI (blue color). 

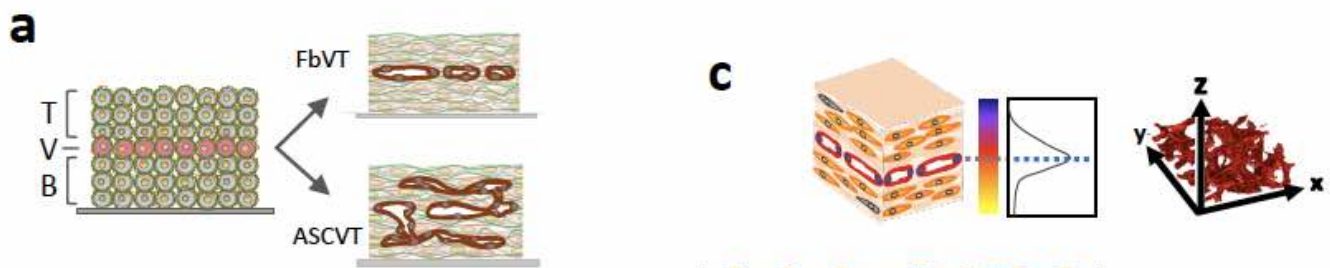

\section{FbVT}
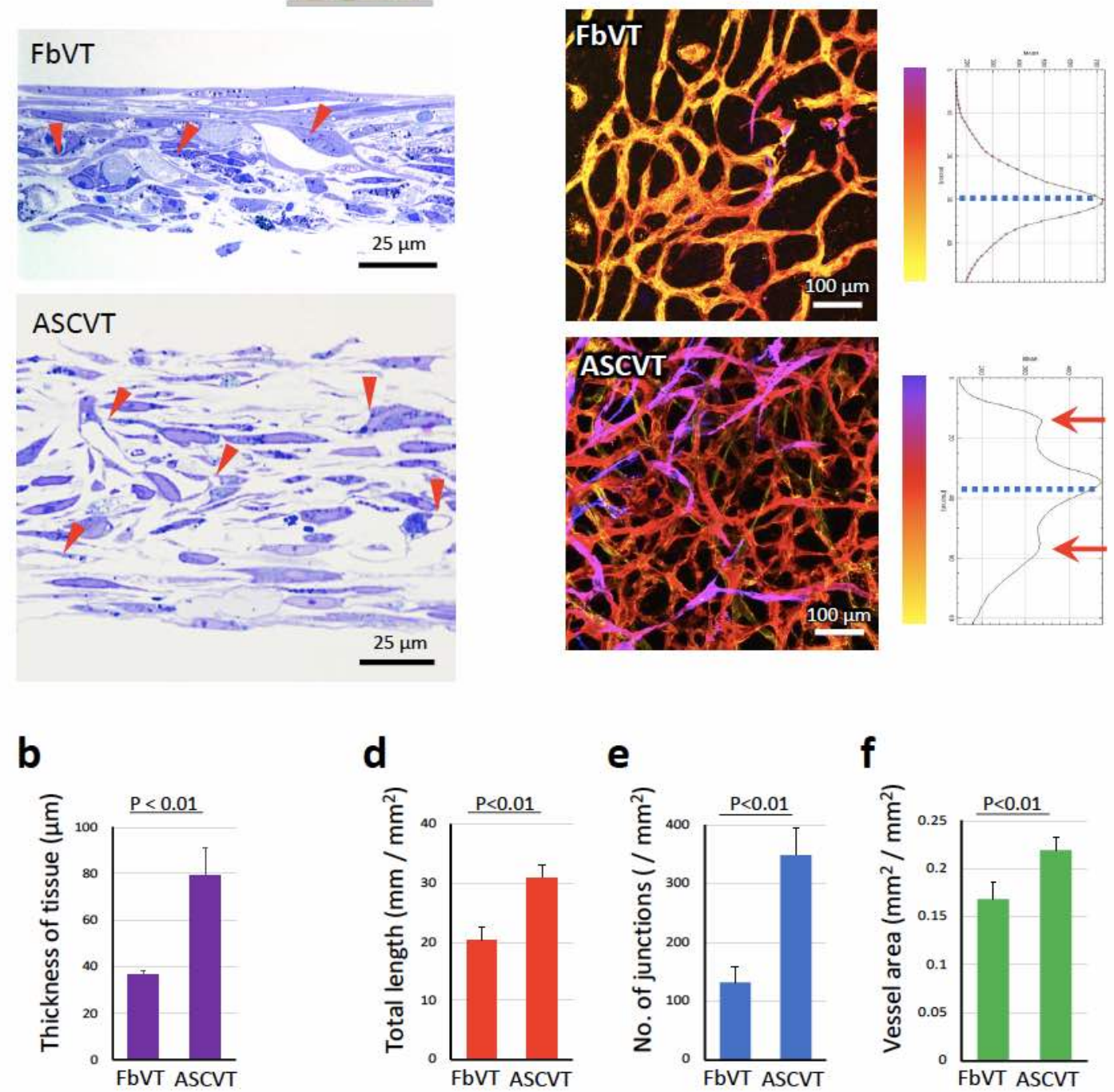

Fig.1 


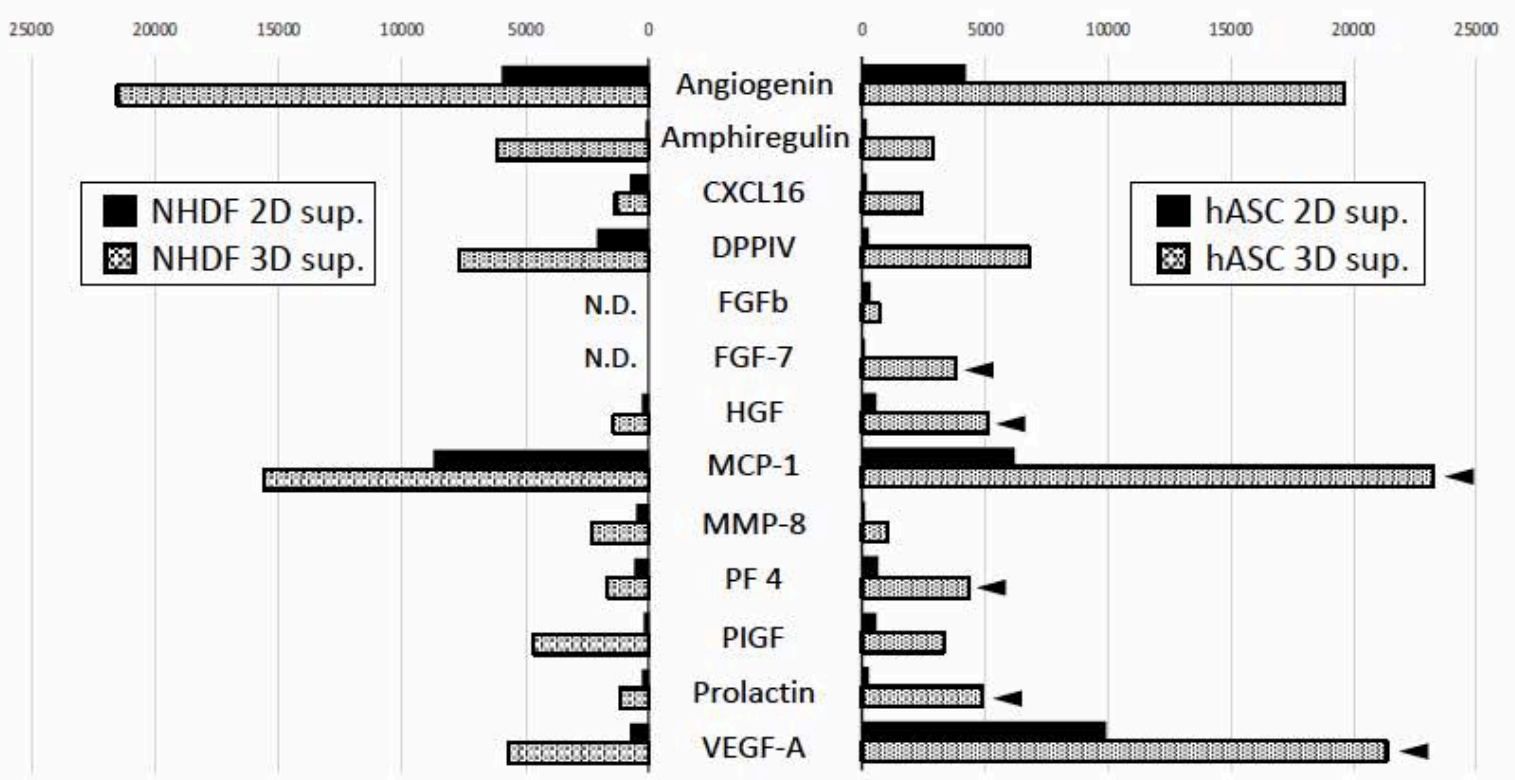

Fig.2 

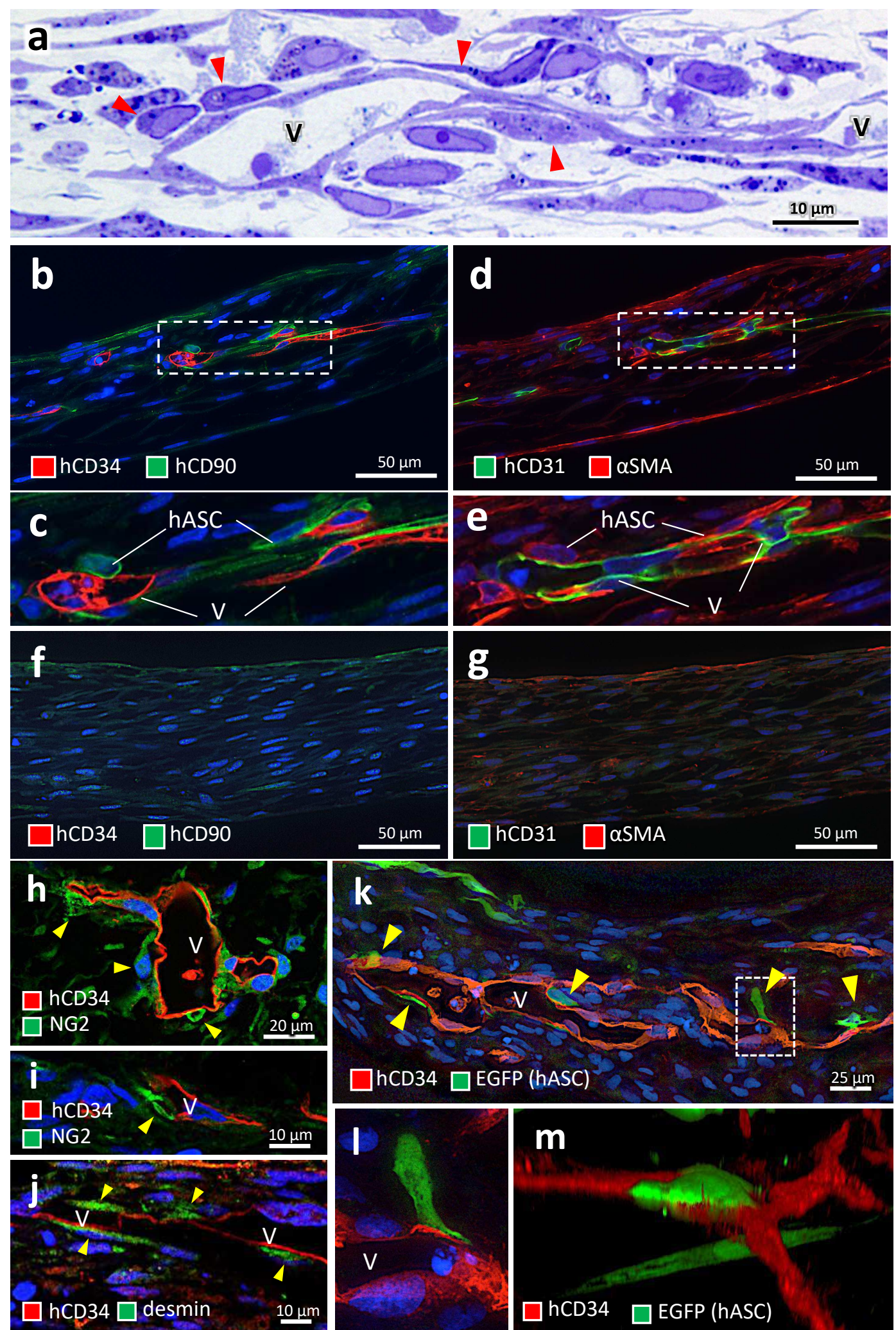

Fig.3 


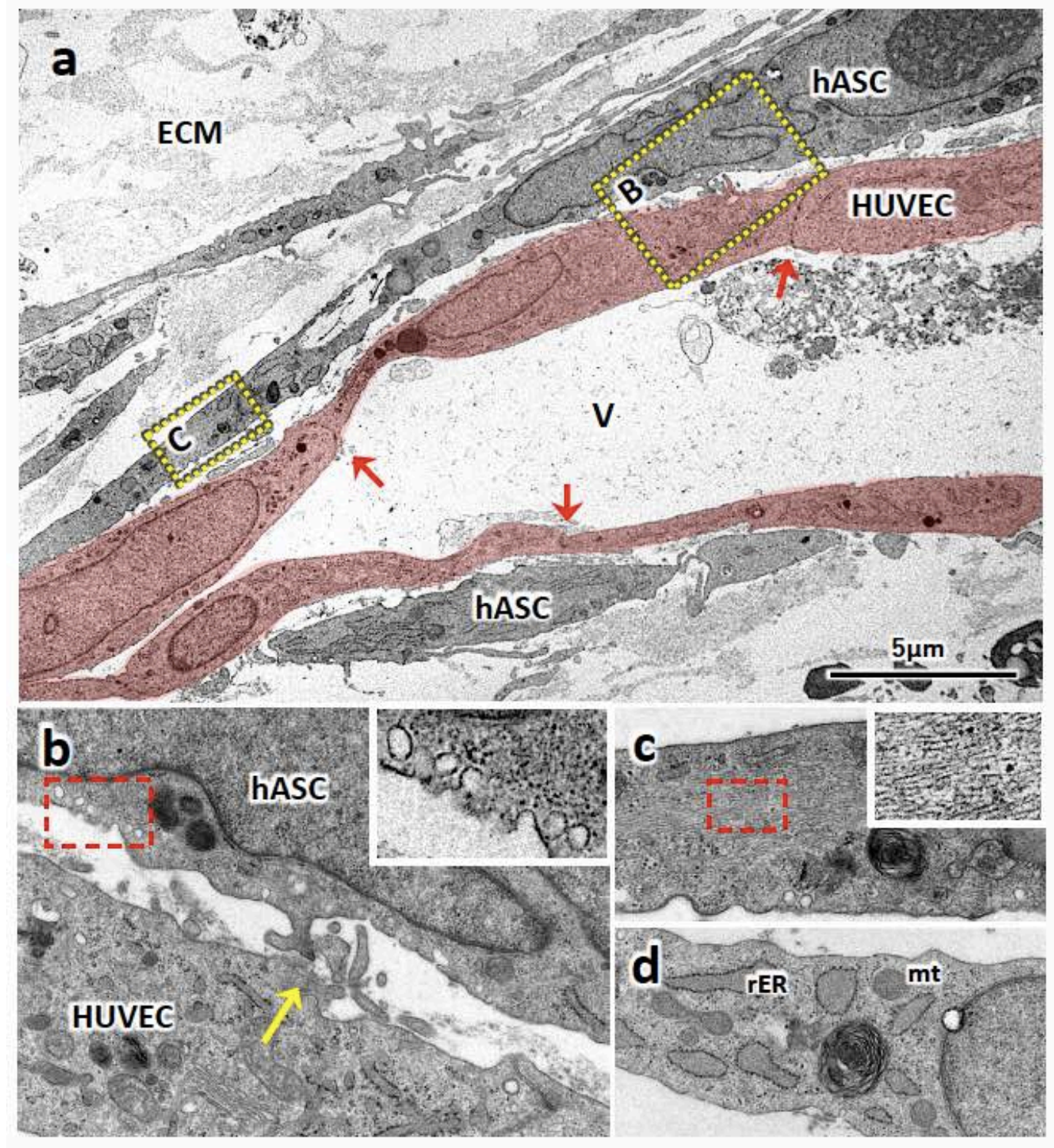

Fig.4 

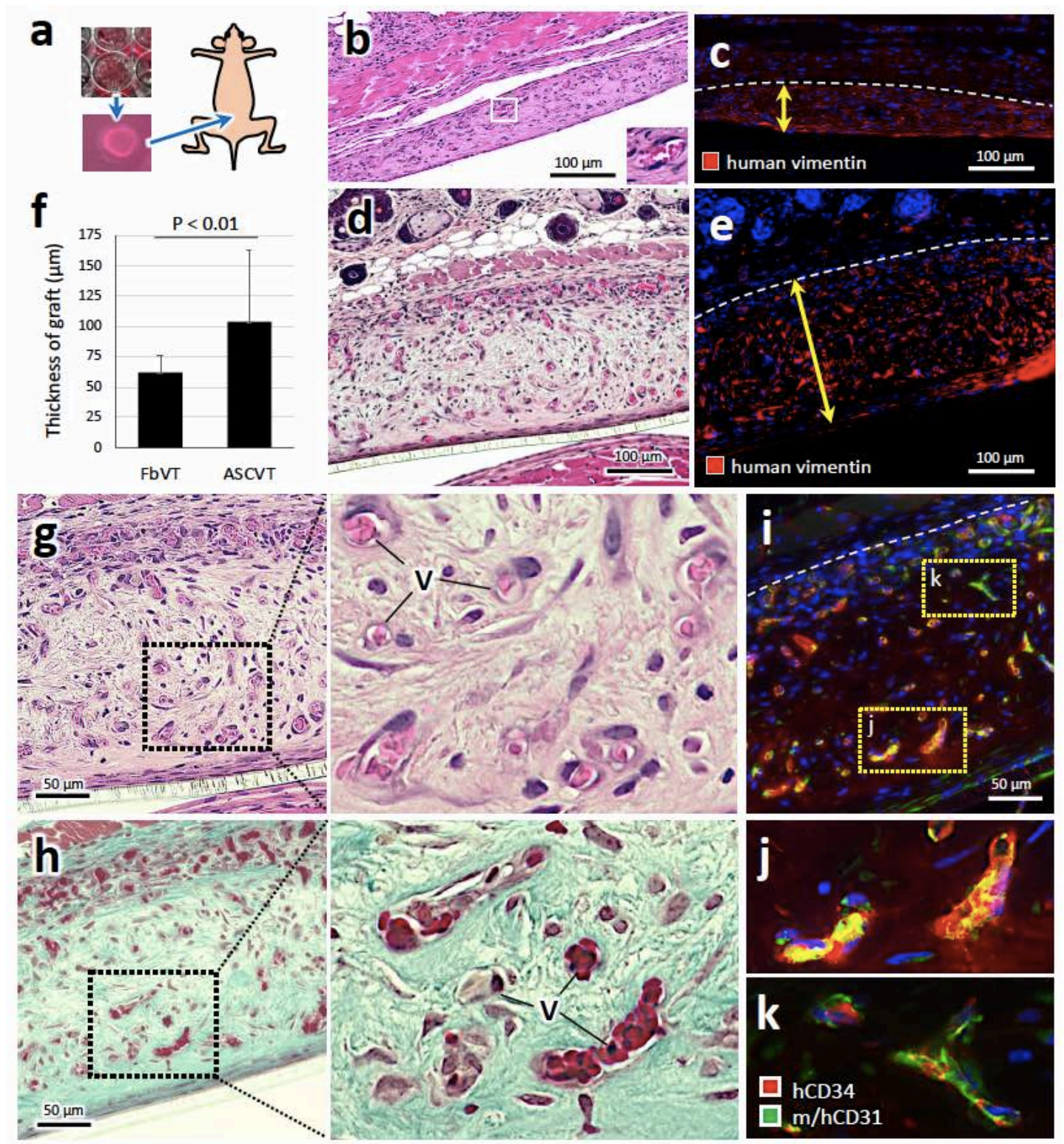

Fig.5 

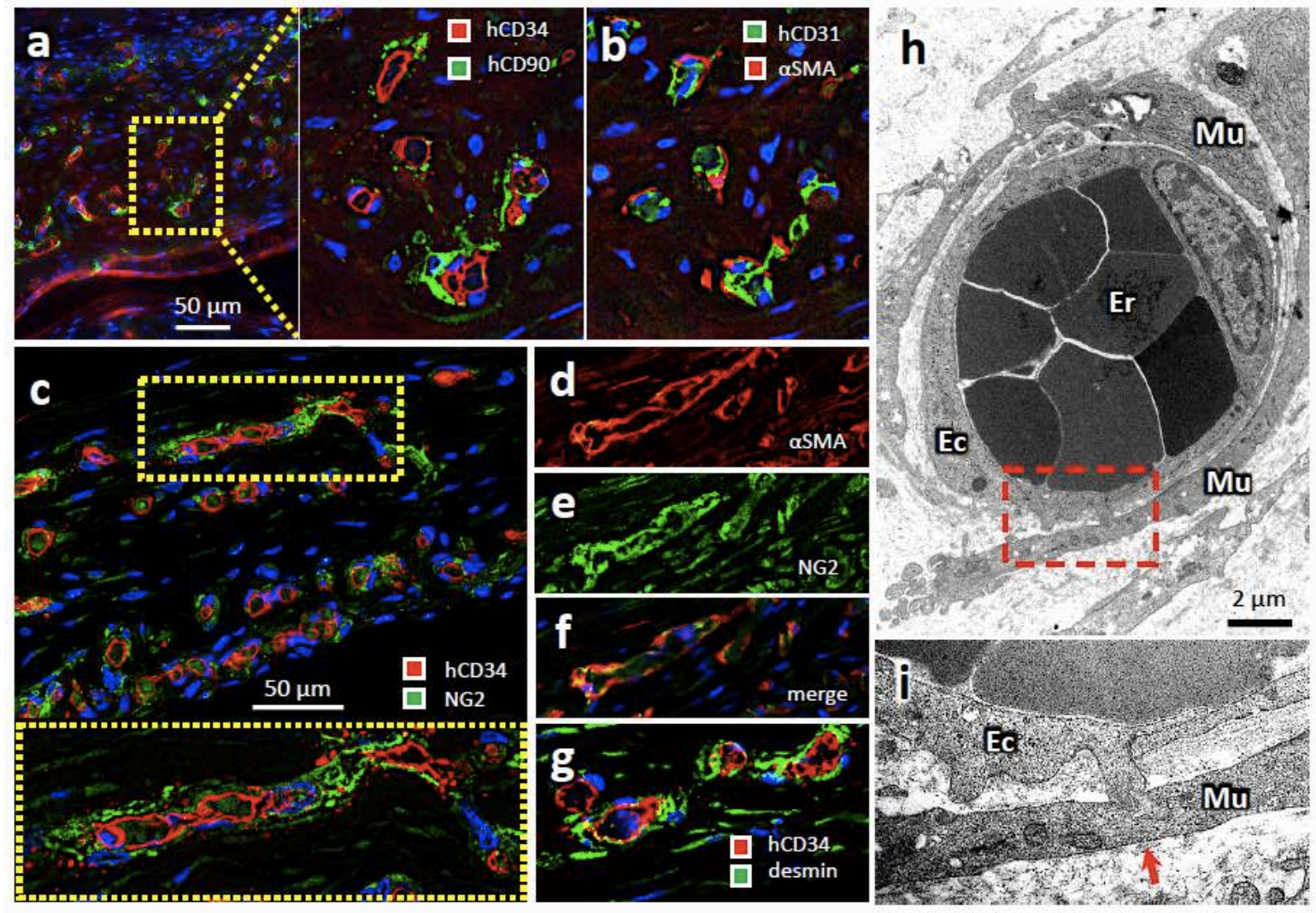

Fig.6 

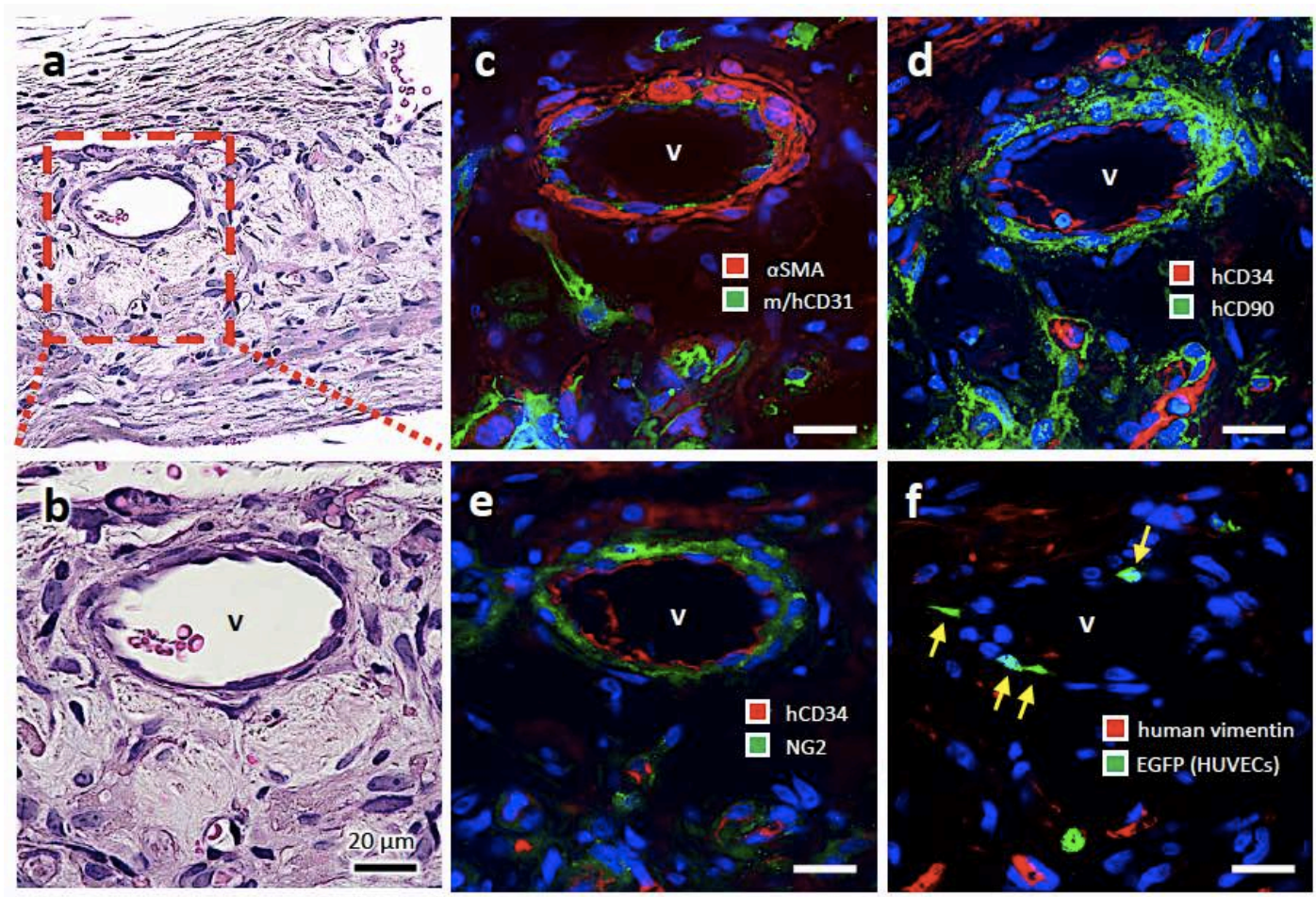

Fig.7 


\section{Figures}

a

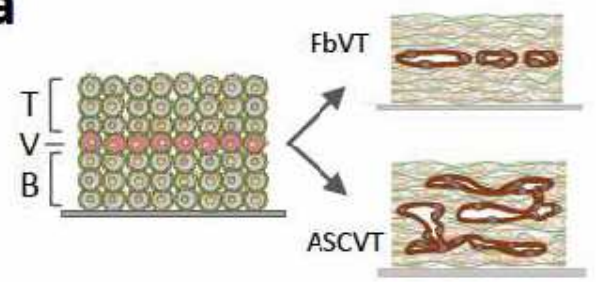

\section{FbVT}
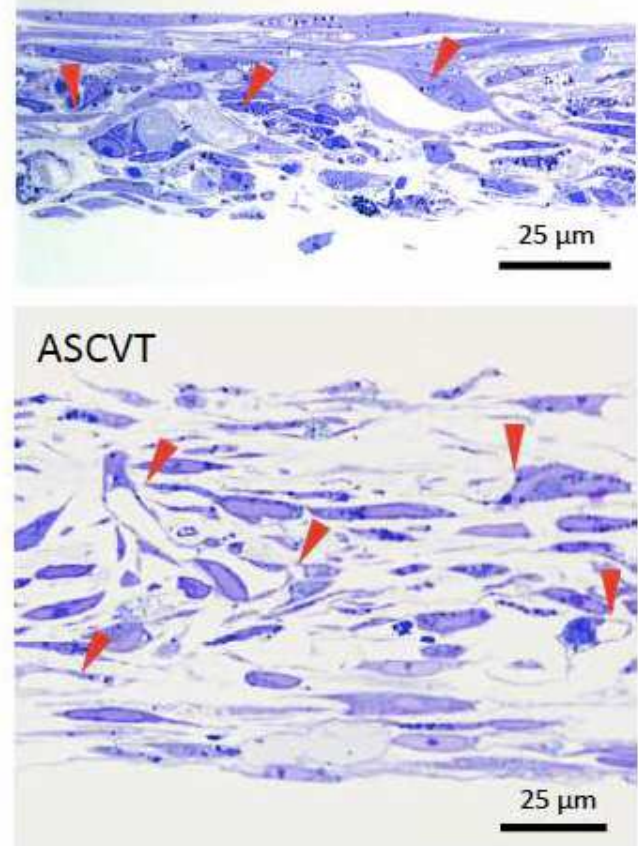

b

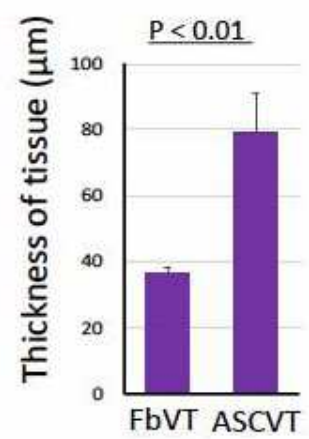

d

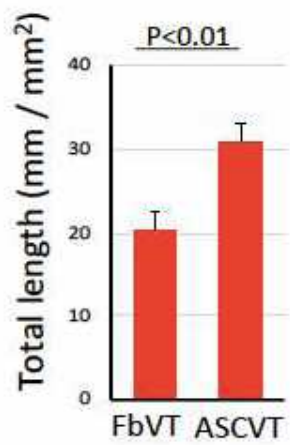

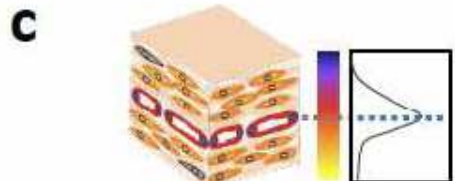
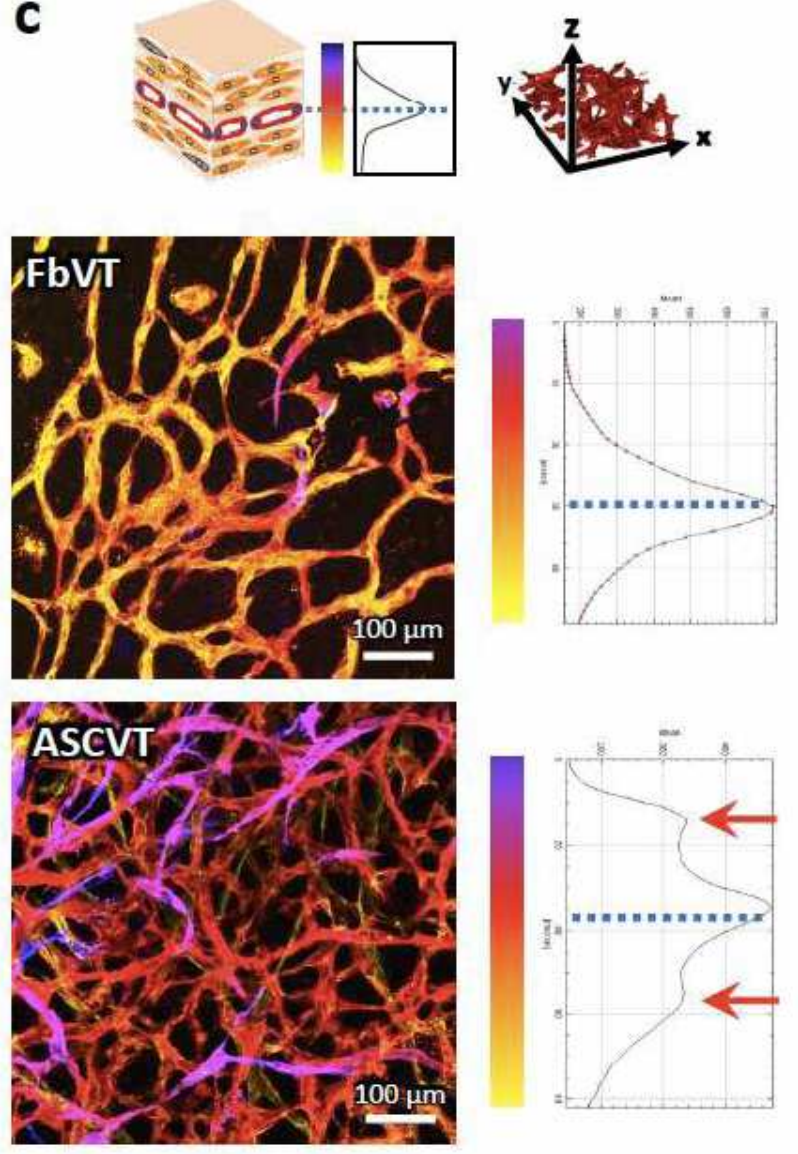

e

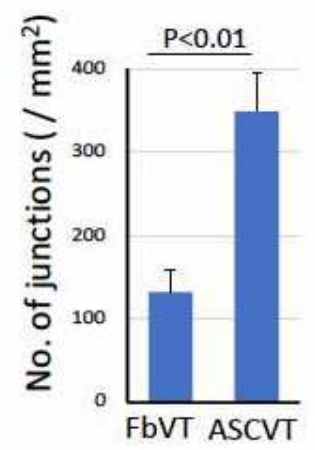


resulted the formation of artificial tissues with vascular structures. The microscopic images of toluidine blue staining sections are shown (FbVT: vascular tissue based on NHDFs; ASCVT: vascular tissue based on hASCs). Arrowheads indicate the distribution of the vascular structures. b: Comparison of the thickness of FbVT and ASCVT. c: The integrated confocal 3D images of human CD34- positive vascular networks in FbVT and ASCVT. Color bars denote the depth along z-axis of the tissue, and the vascular images colored according to the depth of the tissues. The graphs show the relative density distribution of the vascular structure along the depth of the FbVT and ASCVT. Hatched lines indicate the depth of middle vascular layer. The vascular network of ASCVT showed a more dense structure compared to that of FbVT, and spreads to the top and bottom layers indicated by multiple color of the vascular images. Accordingly, the additional peaks of vascular distribution appeared at top and bottom layers as indicated by red arrows. d - $f$ : Quantitative analysis of vascular total length (d), number of junctions (e), and vessel area (f) in FbVT and ASCVT.

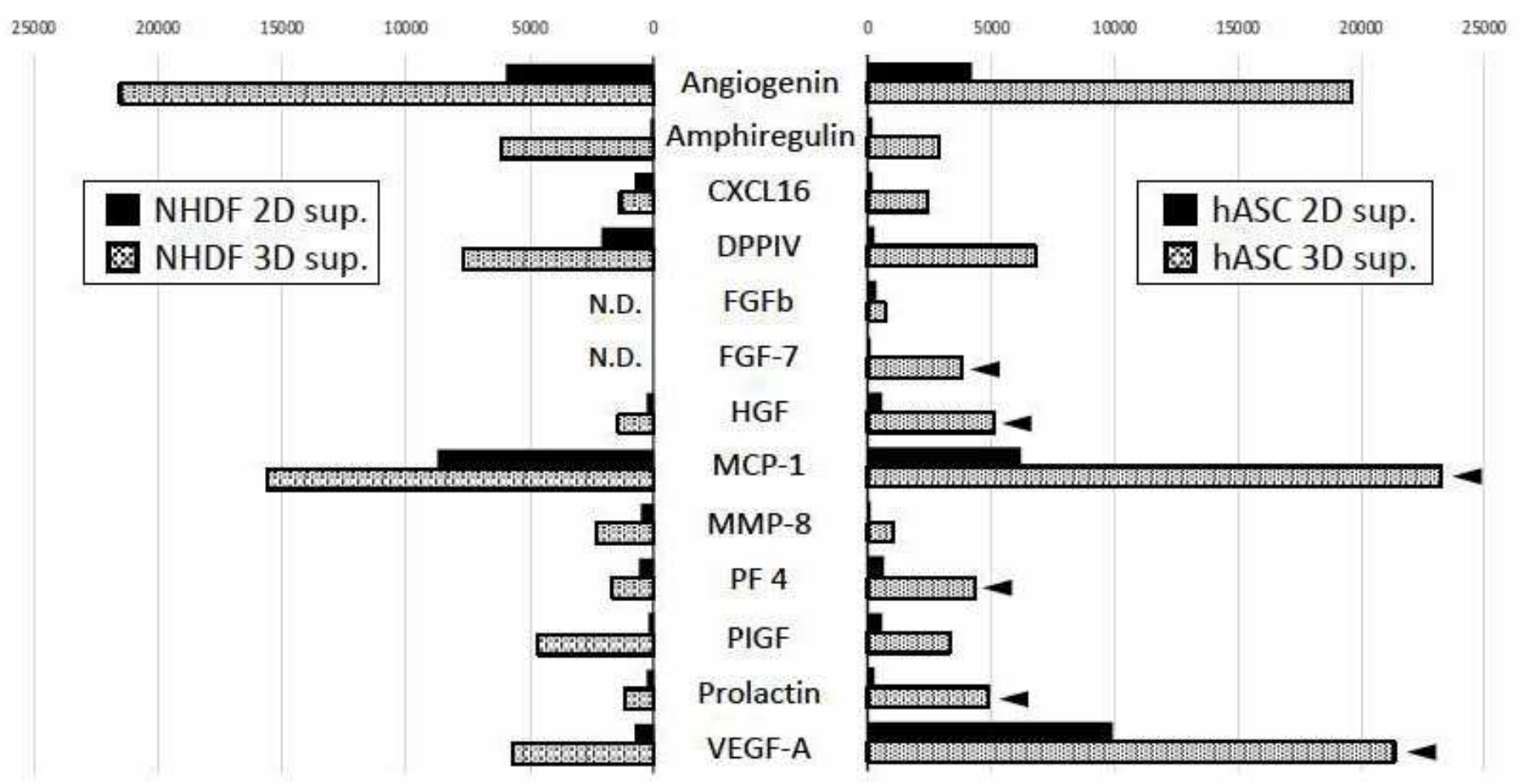

Figure 2

Profiles of angiogenesis-related factors in culture supernatants collected from 2D- and 3D-cultivated NHDFs and hASCs The culture supernatants were analyzed using Proteome Profiler Human Angiogenesis Array Kit (R\&D Systems). Among 55 angiogenesis-related factors, 13 factors increased in 3D cultivation compared to 2D cultivation of NHDFs or ASCs and are shown in these graphs (ND: not detected). Arrowheads: the factors specifically increased in the supernatant from 3D cultivation of ASCs. 


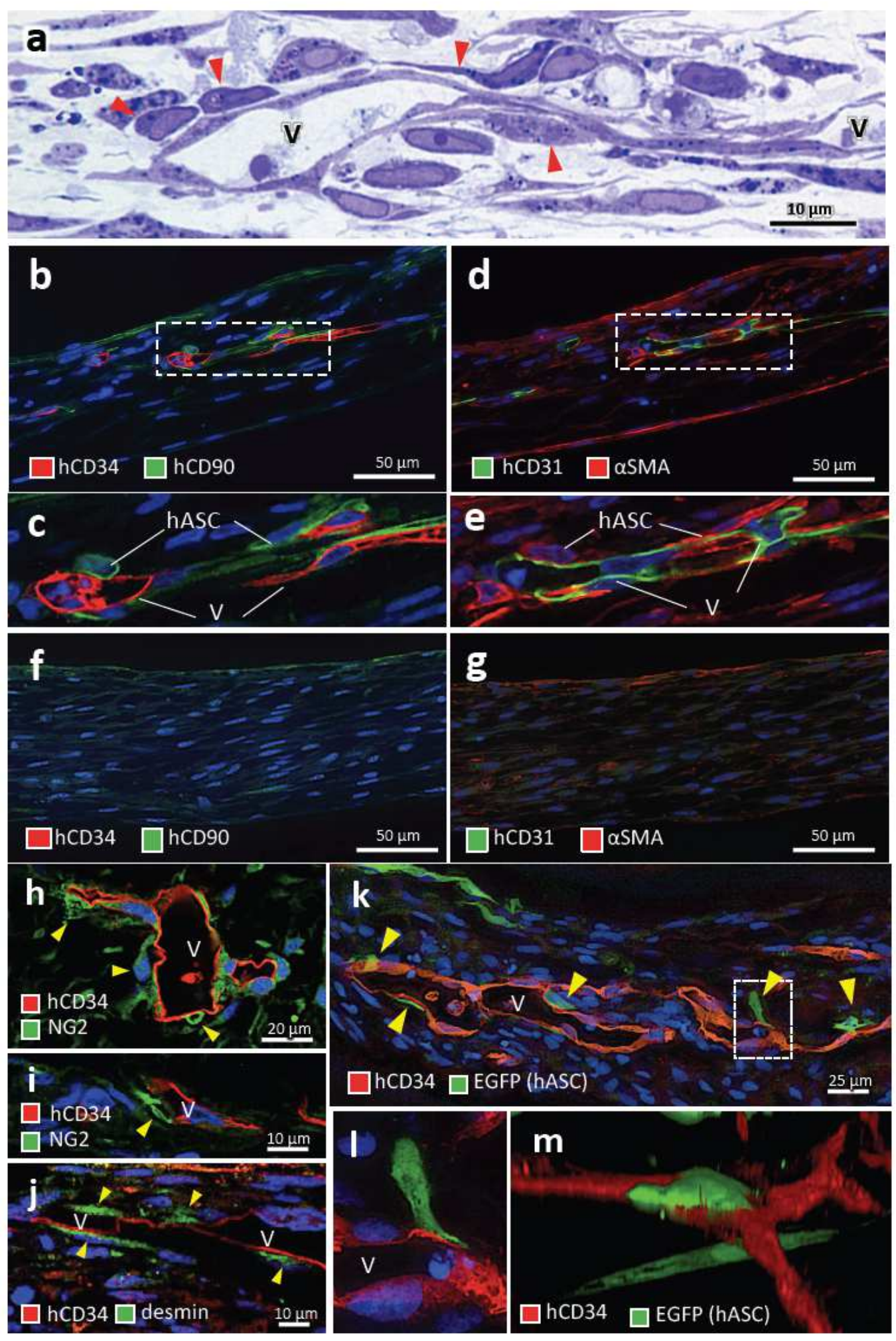

\section{Figure 3}

Perivascular differentiation of hASCs in ASCVT. a: Highly magnified image of epon-embedded and sectioned ASCVT with toluidine blue staining. V: vascular structures. Perivascularly localized hASCs are observed (red arrowheads). b - g: Immunostaining for human CD34 (hCD34) / human CD90 (hCD90) (b, c, f) and human CD31 (hCD31) / aSMA (d, e, g). b - e: ASCVT. Vascular structures (V) are immunostained for hCD34 and hCD31. Perivascularly localized hASCs show intense immunostaining for hCD90 and 
aSMA ( $C$ and $E$ ). $f$ and g: Artificial tissue constructed by hASCs without HUVECs. No vascular structures are found. A weak staining of hCD90 and aSMA is observed. $h$ and $i$ : Perivasculaly localized hASCs showing immunostaining for NG2 (yellow arrowheads). j: Perivasculaly localized hASCs showing immunostaining for desmin (yellow arrowheads). $\mathrm{k}$ - $\mathrm{m}$ : Addition of EGFP-labeled hASCs to fibroblastbased vascular tissue (NHDFs: hASCs: HUVECs = 7: 1: 1). k: Immunostaining of the paraffin section. hASCs with EGFP localized to perivascular area (yellow arrowheads). I: Highly magnified image of hatched box in k. EGFP-positive hASC connected to the vascular structure (V). m: 3D-constructed confocal image. EGFP-positive hASC surrounded the vascular structure like a pericyte. The nuclei of the cells were visualized by DAPI (blue color).

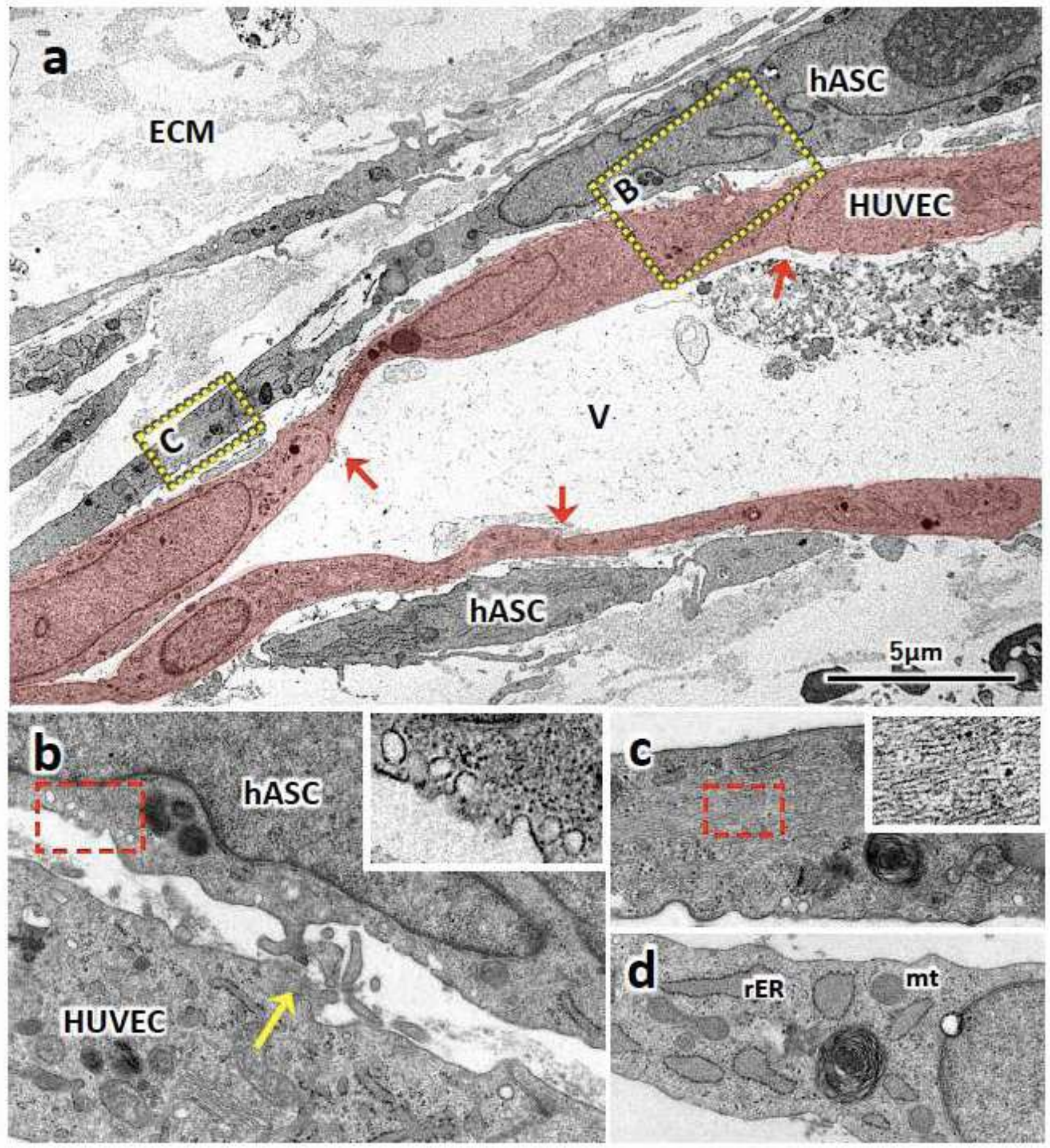




\section{Figure 4}

Ultrastructure of perivascularly localized hASCs in ASCVT a: Electron micrograph of ASCVT. V: vascular structure constructed by HUVECs (redcolored). Interendothelial connecting junctions are observed (red arrow). Perivascularly localized hASCs are also shown. ECM: extracellular matrix is more abundant compared to FbVT in Figure $1 \mathrm{f}$. b: High magnified image of hatched box in a. The projections from hASC interact with HUVEC, suggesting the formation of peg-socket structure (yellow arrow). Inset shows further high magnified image of red hatched box containing caveola at cytoplasmic membrane. c: Another high magnified image of hatched box in a. The cytoplasm of perivascular hASCs shows filamentous structure that is considered as presence of actin filaments. Inset shows further high magnified image of red hatched box on cytoplasm. d: The image of hASCs locate far from vascular structures. The cytoplasm shows the fibroblast-like structure with abundant rough endoplasmic reticulum (rER) and mitochondria $(\mathrm{mt})$. 


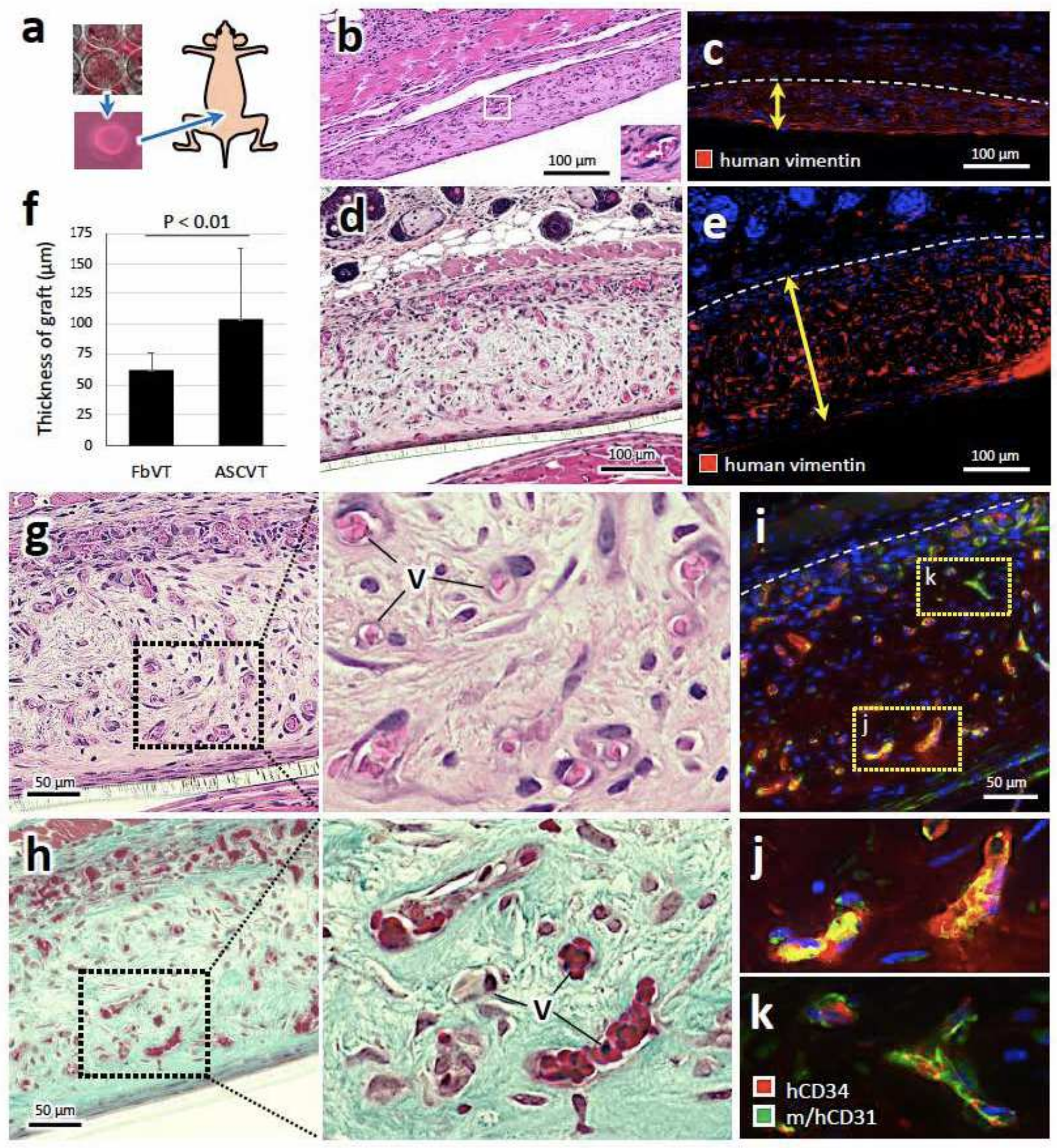

\section{Figure 5}

Subcutaneous transplantation of ASCVT to nude mice a: FbVT and ASCVT were cut out from the Transwell inserts and subcutaneously transplanted to back skin of nude mice. $b$ - e: engrafted FbVT ( $b$ and c) and ASCVT ( $d$ and e) at two weeks after the transplantation. b and d: HE staining; $c$ and e: immunostaining for human vimentin. The FbVT engrafts as connective tissue containing vascular networks with host blood (b, inset). The thickness of engrafted human-derived tissue is visualized by 
immunoreaction of human vimentin (c). The ASCVT also subcutaneously engrafted with vascular networks (d), and the thickness was higher than engrafted FbVT (e). f: Quantitative comparison of the graft thickness between FbVT and ASCVT. The ASCVT significantly shows higher thickness. $g$ - $k$ : High magnified images of engrafted ASCVT. g: HE staining. The graft contains the capillary- or small venulelike structures with host blood $(V)$ and abundant stroma. h: Masson Goldner staining for serial section of $\mathrm{g}$. The stroma stained for collagen fibers, indicating the construction of connective tissue. $\mathrm{i}-\mathrm{k}$ : Immunostaining by using antibodies for human CD34 (red) and mouse / human CD31 (green). The engrafted vessels derived from HUVEC are positive for both antibodies ( $i$ and $j$ ). At the peripheral area of the graft, the vessels with staining by antibodies for mouse / human CD31 and partial staining by antibodies for human CD34 are observed ( $i$ and $k$ ) suggesting the anastomotic region between host and graft circulation. The nuclei of the cells in the dark field images were visualized by DAPI (blue color).
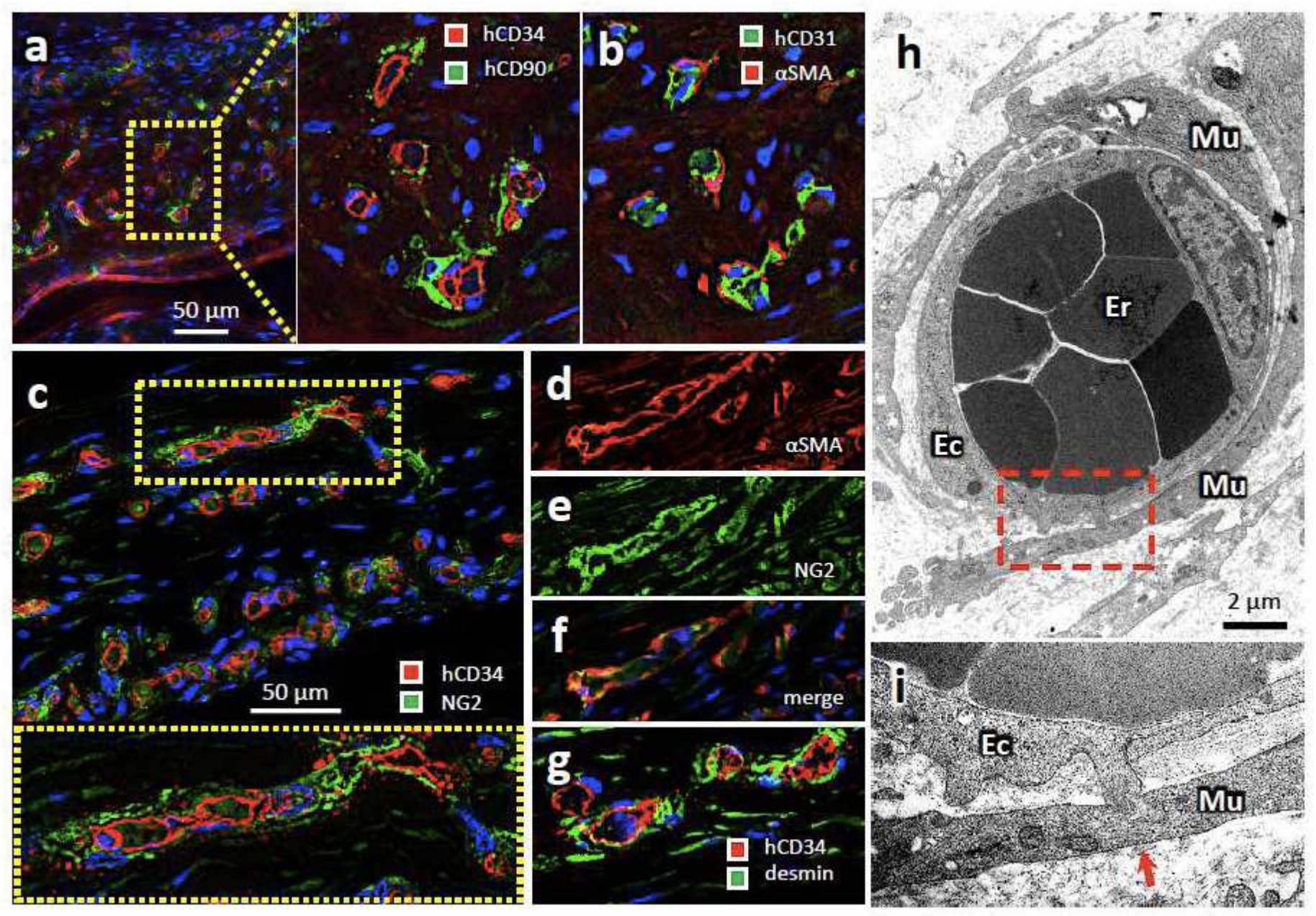

Figure 6

The vascular mural structure in the engrafted ASCVT at 2 weeks after the transplantation a: Immunostaining for human CD34 (hCD34) and human CD90 (hCD90). Almost every engrafted and hCD34-positive vessels surrounded by hCD90-positive mural cells that putatively derived from hASCs. b: Immunostaining for human CD31 (hCD31) and aSMA in serial section of $A$. The endothelial cells are positive for hCD31 in addition to hCD34. The mural cells are positive for aSMA. c: Immunostaining for 
hCD34 and NG2. The mural cells are positive for NG2 that is a marker of pericytes and vascular smooth muscle cells. $\mathrm{d}$ - $\mathrm{f}$ : double immunostaining for aSMA and NG2. The merged image indicated coexistence of aSMA and NG2 in the mural cells derived from hASCs. g: The presence of desmin, another marker of pericytes and smooth muscle cells, is confirmed in the mural cells. h: electron micrograph of the vessels in engrafted ASCVT. Er: erythrocytes; Ec: endothelial cells; Mu: mural cells. Stratifying mural cells surrounded the vessels. i: High magnified image of hatched box in h. Formation of peg-socket structure between endothelial cell and the mural cell is found (arrow). The nuclei of the cells in the dark field images were visualized by DAPI (blue color).
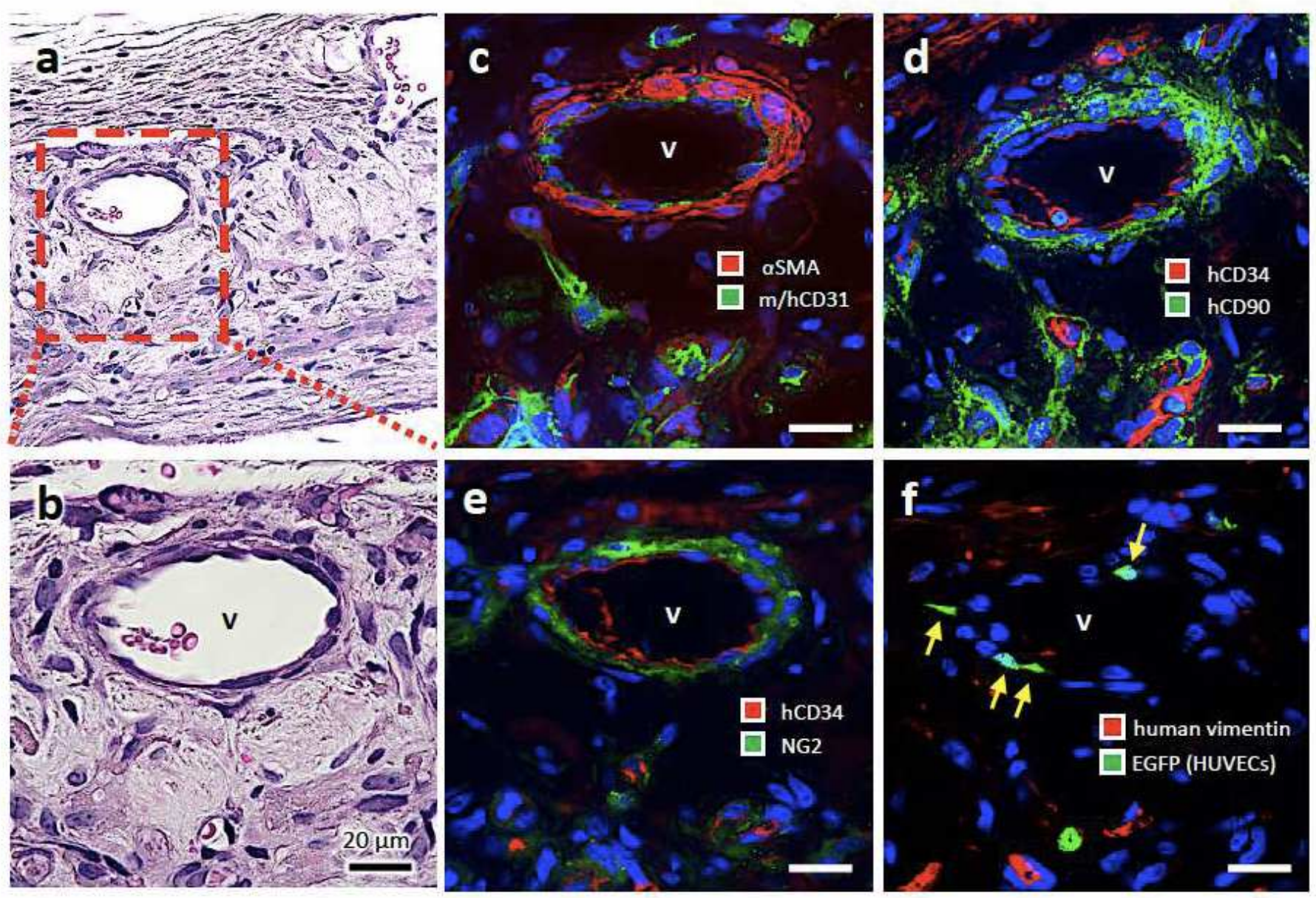

\section{Figure 7}

The blood vessels in the engrafted ASCVT at four weeks after the transplantation a - $\mathrm{f}$ : The engrafted ASCVT at four weeks after transplantation. The serial sections are used for each staining. $a$ and $b$ : HE staining. Enlarged vein-like vessels $(\mathrm{V})$ are found in the graft. $\mathrm{c}-\mathrm{f}$ : Immunostaining. The endothelium of vein-like vessel is positive for both mouse / human CD31 (m/hCD31) (c) and human CD34 (hCD34) (d and e). The mural cells show positive immunostaining for aSMA (c), human CD90 (hCD90) (d), and NGs (e), indicating that this structure derived from hASCs. f: Immunostaining for human vimentin and EGFP. The engrafted ASCVT was constructed by using HUVEC labeled by EGFP. The endothelial cells showed EGFP-positive immunostaining (arrows), demonstrating that the vascular structures in ASCVT engrafted 
and constructed the vein-like vessels surrounded by mural cells. The nuclei of the cells in the dark field images were visualized by DAPI (blue color).

\section{Supplementary Files}

This is a list of supplementary files associated with this preprint. Click to download.

- 201231suppldata.pdf 